\title{
ALTERNATING SIGN AND SIGN-RESTRICTED MATRICES: REPRESENTATIONS AND PARTIAL ORDERS*
}

\author{
RICHARD A. BRUALDI ${ }^{\dagger}$ AND GEIR DAHL ${ }^{\ddagger}$
}

\begin{abstract}
Sign-restricted matrices (SRMs) are $(0, \pm 1)$-matrices where, ignoring 0's, the signs in each column alternate beginning with $\mathrm{a}+1$ and all partial row sums are nonnegative. The most investigated of these matrices are the alternating sign matrices (ASMs), where the rows also have the alternating sign property, and all row and column sums equal 1 . We introduce monotone triangles to represent SRMs and investigate some of their properties and connections to certain polytopes. We also investigate two partial orders for ASMs related to their patterns alternating cycles and show a number of combinatorial properties of these orders.
\end{abstract}

Key words. Sign-restricted matrices, Alternating sign matrices, Monotone triangles, Polytopes, Partial order.

AMS subject classifications. 05B20, 15B48.

1. Introduction. Matrices with constraints on the sign of entries, or the sum of entries, are of interest in many settings. A sign-restricted matrix, or SRM, is an $m \times n(0, \pm 1)$-matrix $A$ such that each partial column sum, starting from row 1 , equals 0 or 1 , and each partial row sum, starting from column 1 , is nonnegative, see $[11,17]$. The constraint on each column implies that its nonzeros alternate, starting with a +1 . This notion was introduced in [1] and it was shown that this class is in bijection with so-called semistandard Young tableaux (see also [17]). An important subclass of SRMs is the alternating sign matrices (ASMs); these are square SRMs where both the rows and columns are alternating starting and ending with a +1 , see $[2,3,6,8,9,10,18]$. We let $\mathcal{S}_{m, n}$ denote the class of $m \times n$ SRMs, abbreviated to $\mathcal{S}_{n}$ if $m=n$, and we let $\mathcal{A}_{n}$ denote the class of $n \times n$ ASMs. ASMs arise in the study of crystals in physics. Examples of ASMs are the permutation matrices; these are the ASMs without any -1 's. More generally, a $(0,1)$-matrix with at most one 1 in every column is an SRM. Row 1 and column 1 of an SRM can only contain 0s and +1 s and, in particular, column 1 can contain only one +1 . The transpose of an ASM is another ASM, while the transpose of an SRM need not be an SRM.

Our main goal is to continue the study of SRMs and ASMs and introduce some new notions of interest for these matrix classes. The main contributions of this paper can be summarized as follows:

(i) It is known that monotone triangles represent ASMs (as discussed in section 2). We generalize this notion to SRMs, and call them pseudo-monotone triangles. As with ASMs, we use the notion of the partial-column-sums matrix. We show some properties of pseudo-monotone triangles in Section 3. We also determine the maximum number of nonzero rows in an SRM in the case that each column sum is 1 .

(ii) Section 4 considers related polytopes, and we determine the convex hull of partial-column-sums matrices of permutation matrices, and also the convex hull of all monotone triangles.

${ }^{*}$ Received by the editors on August 5, 2021. Accepted for publication on September 3, 2021. Handling Editor: Zejun Huang. Corresponding author: Geir Dahl.

${ }^{\dagger}$ Department of Mathematics, University of Wisconsin, Madison, WI 53706, USA (brualdi@math.wisc.edu).

¥Department of Mathematics, University of Oslo, Norway (geird@math.uio.no). 
(iii) Section 5 considers other notions and questions concerning SRMs and ASMs. An expansion of an SRM is to replace some zeros by nonzeros in such a way that a new SRM is obtained, and deflation is the opposite operation. This leads to two different partial orders on the class of ASMs and we establish a number of results on these orders in this section and the two final Sections 6 and 7 . In particular, we study minimal and maximal elements and relationships between the orders.

2. Monotone triangles and ASMs. In this section, we review briefly the equivalence of ASMs and monotone triangles.

A triangle $T$ of order $n$ is a triangular array of integers with $n$ rows. For example, with $n=5$, we have

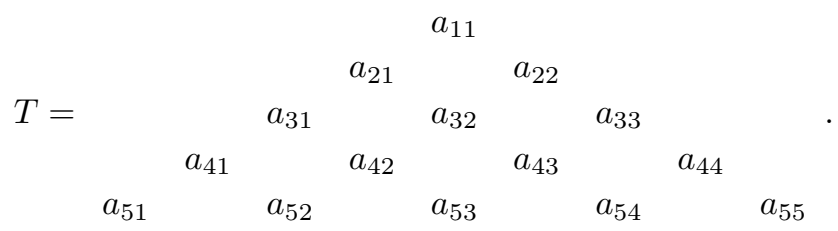

By left-justifying the entries, we may also represent the triangle $T$ by an $n \times n$ lower triangular matrix $L_{T}$. Thus, $T$ in (2.1) becomes

$$
L_{T}=\left[\begin{array}{l|l|l|l|l}
a_{11} & & & & \\
\hline a_{21} & a_{22} & & & \\
\hline a_{31} & a_{32} & a_{33} & & \\
\hline a_{41} & a_{42} & a_{43} & a_{44} & \\
\hline a_{51} & a_{52} & a_{53} & a_{54} & a_{55}
\end{array}\right] .
$$

The rows of $T$ are the rows of $L_{T}$; the descending diagonals of $T$ are the descending diagonals of $L_{T}$, and the ascending diagonals of $T$ are the ascending columns of $L_{T}$. $L_{T}$ can also be obtained from $T$ by rotating its ascending diagonals by 90 degrees in a counterclockwise direction. We generally depict triangles as lower triangular matrices with empty positions as in (2.2).

A triangle $T$ of order $n$ is a monotone triangle $[12,15]$, abbreviated to MT, provided its entries are from the set $\{1,2, \ldots, n\}$ and it has the properties that the entries are strictly increasing along the rows from left to right, and weakly increasing on both the ascending and descending diagonals. Viewed as the lower triangular matrix $L_{T}$, an MT has the following properties:

(a) the rows are strictly increasing from left to right,

(b) the columns are weakly increasing from bottom to top, and

(c) the descending diagonals are weakly increasing from left to right.

The last row of an MT is $1,2, \ldots, n$. In the case of $n=5$, we have the following example in both forms:

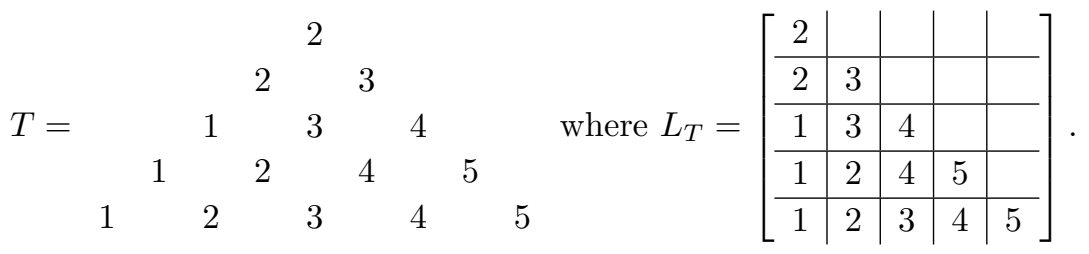


Electronic Journal of Linear Algebra, ISSN 1081-3810

A publication of the International Linear Algebra Society

Volume 37, pp. 613-639, September 2021.

615

Alternating sign and sign-restricted matrices: Representations and partial orders

Monotone triangles arise in the study of alternating sign matrices (ASMs) [14] which are now reviewed. The partial-column-sums matrix, abbreviated to PCSM, of an $m \times n$ matrix $A=\left[a_{i j}\right]$ is the $m \times n$ matrix $A^{*}=\left[a_{i j}^{*}\right]$ where $a_{i j}^{*}=\sum_{k=1}^{i} a_{k j}$, the sum of the entries in column $j$ of $A$ down to row $i$. For example, an $\operatorname{ASM} A$ is given below along with its $\operatorname{PCSM} A^{*}$ :

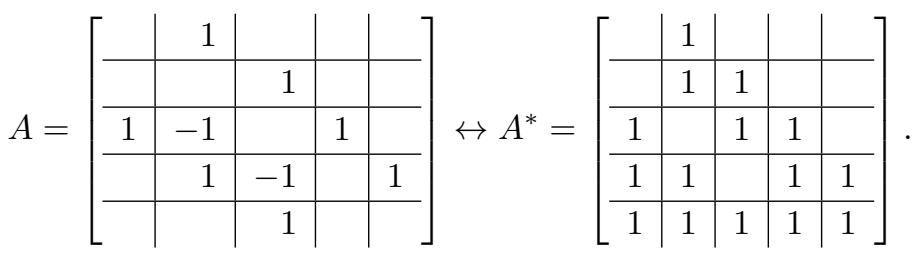

(We usually denote a zero in a position by leaving the position empty.) As in the example the alternating property of the rows and columns implies that the PCSM of an ASM is a $(0,1)$-matrix with row sums $1,2, \ldots, n$. As shown below, replacing the 1 's by the column number in which they appear gives the matrix $A^{* *}$ of column numbers of the 1 's of $A^{*}$ resulting in the corresponding monotone triangle:

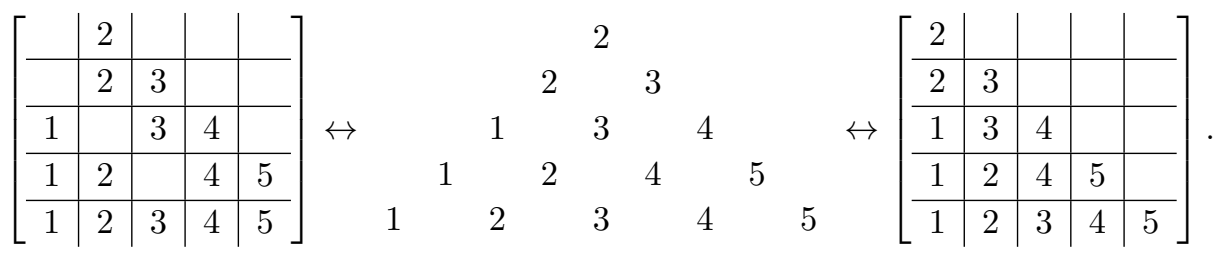

As seen in this example, our way to denote an MT amounts to left-justifying the nonzeros of the matrix $A^{* *}$ of column numbers giving a classical Ferrers diagram. The set $\mathcal{A}_{n}$ of $n \times n$ ASMs is in bijective correspondence with the set $\mathcal{M}_{n}$ of monotone triangles of order $n$. The arrows are in both directions since it is easy to reverse the constructions

$$
A \rightarrow A^{*} \rightarrow A^{* *} \rightarrow T \rightarrow L_{T}
$$

In summary, via its PCSM, an $n \times n$ ASM $A$ uniquely corresponds to an $n \times n(0,1)$-matrix $A^{*}$ with $k 1$ 's in row $k$ for $k=1,2, \ldots, n$ and, by replacing the 1 's of $A^{*}$ with their column numbers to get $A^{* *}, A^{* *}$ uniquely corresponds to a MT in either one of our two forms.

Permutation matrices $P$ being ASMs give MTs from which the corresponding permutation can be easily read off: the PCSM matrix of an $n \times n$ permutation matrix corresponds to a saturated chain in the partially ordered set of subsets of $\{1,2, \ldots, n\}: S_{0} \subset S_{1} \subset S_{2} \subset \cdots \subset S_{n}$ where $\left|S_{i}\right|=i$ for $i=1,2, \ldots, n$. For example, the permutation $\pi=(3,1,4,2,5)$ gives:

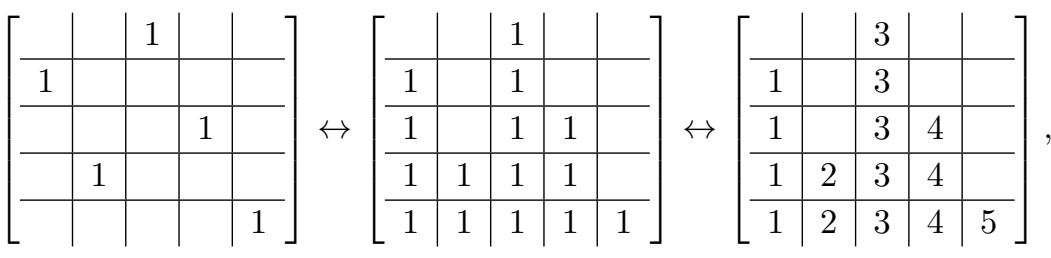


and then

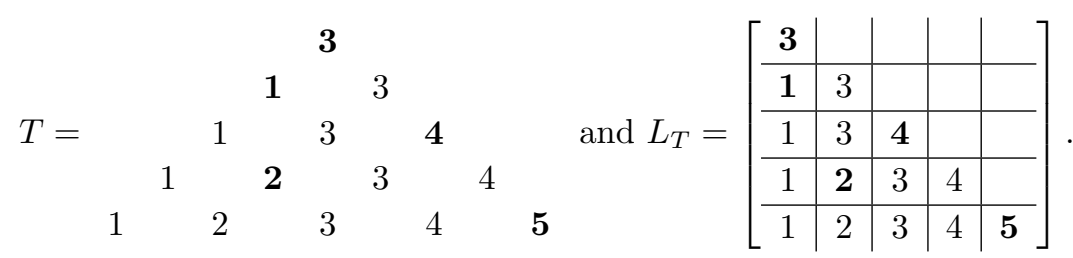

First occurrences from top to bottom of 1, 2, 3, 4, 5 are in boldface and give the permutation $\pi$. This property of the PCSM of a permutation matrix is not generally satisfied by ASMs. It is reflected in the corresponding MT by the property that the integers in row $i$ are contained in the integers of row $i+1$ $(1 \leq i \leq n-1)$.

3. Pseudo-monotone triangles and SRMs. We consider the construction of a partial-column-sums matrix and a monotone triangle for sign-restricted matrices (SRMs) obtaining what we call a pseudomonotone triangle, abbreviated to PMT. Recall that an SRM is an $m \times n(0, \pm 1)$-matrix such that all partial-column-sums (from top to bottom) are 0 or 1 , and all partial row sums (from left to right) are only nonnegative. Without any real loss of generality, we may assume that the full column sums of an SRM all equal 1, as with ASMs, since otherwise we may include additional rows at the bottom with all 0's except for a single 1 . We call these normalized $S R M \mathrm{~s}$. Recall that $\mathcal{S}_{m, n}$ denotes the set of $m \times n$ SRMs. Let $\mathcal{S}_{m, n}^{+}$ denote the set of $m \times n$ normalized SRMs. We also denote by $\mathcal{S}_{m, n}^{++}$the set of normalized SRMS without any -1 's (so exactly one 1 in every column). Then PMTs of matrices in $\mathcal{S} \mathcal{R}_{m, n}^{++}$correspond to chains of length $m$

$$
S_{0}=\emptyset \subseteq S_{1} \subseteq S_{2} \subseteq \cdots \subseteq S_{m}=\{1,2, \ldots, n\},
$$

in the partially ordered set of subsets of $\{1,2, \ldots, n\}$ with repeats allowed.

With an SRM $A$, we can associate a PCSM using the same construction as with an ASM. Because the partial-column-sums of an SRM are 0 or 1, its PCSM is also a (0,1)-matrix. Then the PMT corresponding to $A$ is obtained from the PCSM exactly as the MT in the case of an ASM (but it need not be a triangle) giving a left-justified diagram whose set of entries is $\{1,2, \ldots, n\}$. The last row of an PMT of a normalized $\mathrm{SRM}$ is $1,2, \ldots, n$.

ExAMPLE 3.1. Consider the SRM below and the resulting PCSM and PMT:

$\left[\begin{array}{r|r|r|r} & 1 & & 1 \\ \hline 1 & -1 & 1 & -1 \\ \hline 1 & -1 & 1 \\ \hline & & 1 & \end{array}\right] \leftrightarrow\left[\begin{array}{l|l|l|l} & 1 & & 1 \\ \hline 1 & & 1 & \\ \hline 1 & 1 & & 1 \\ \hline 1 & 1 & 1 & 1\end{array}\right] \leftrightarrow\left[\begin{array}{l|l|l|l} & 2 & & 4 \\ \hline 1 & & 3 & \\ \hline 1 & 2 & & 4 \\ \hline 1 & 2 & 3 & 4\end{array}\right] \leftrightarrow\left[\begin{array}{l|l|l|l}2 & 4 & & \\ \hline 1 & 3 & & \\ \hline 1 & 2 & 4 & \\ \hline 1 & 2 & 3 & 4\end{array}\right]$.

The arrows are in both directions since, as with ASMs, it is easy to reverse these constructions.

The PCSM provides the transition between ASMs and MTs and also between SRMs and PMTs. We now study properties of these matrices in detail.

Let $M_{m, n}$ denote the linear space of real $m \times n$ matrices. Let $S_{m, n}: M_{m, n} \rightarrow M_{m, n}$ be the linear transformation that maps $A=\left[a_{i j}\right] \in M_{m, n}$ into the partial-column-sums matrix $S_{m, n}(A)=A^{*}=\left[a_{i j}^{*}\right] \in$ $M_{m, n}$ where

$$
a_{i j}^{*}=\sum_{k=1}^{i} a_{k j} \quad(i \leq m, j \leq n) .
$$


Electronic Journal of Linear Algebra, ISSN 1081-3810

A publication of the International Linear Algebra Society

Volume 37, pp. 613-639, September 2021.

$S_{m, n}$ is an isomorphism, and its inverse is given by $S_{m, n}^{-1}\left(A^{*}\right)=A$ where

$$
a_{i j}=a_{i j}^{*}-a_{i-1, j}^{*} \quad(i \leq m, j \leq n),
$$

and $a_{0 j}^{*}:=0(j \leq n)$. We use the abbreviations $M_{n}$ and $S_{n}$ in case $m=n$.

Let $A=\left[a_{i j}\right]$ be an $m \times n \mathrm{SRM}$, and let $A^{*}=\left[a_{i j}^{*}\right]=S_{m, n}(A)$ be its PCSM. The property of SRMs that all column sums equal 0 or 1 implies that $S_{m, n}(A)$ is a $(0,1)$-matrix. Let $i \leq m$ and let $r_{i}\left(A^{*}\right)$ be the $i$ 'th row sum in of its PCSM $A^{*}$, that is the number of ones in row $i$ of $A^{*}$. If $A$ is an $\operatorname{ASM}$, then $r_{i}\left(A^{*}\right)=i$ $(i \leq n)$. For normalized SRMs, the property that all row sums are nonnegative implies that

$$
0 \leq r_{1}\left(A^{*}\right) \leq r_{2}\left(A^{*}\right) \leq \cdots \leq r_{m}\left(A^{*}\right)=n
$$

For example, we have

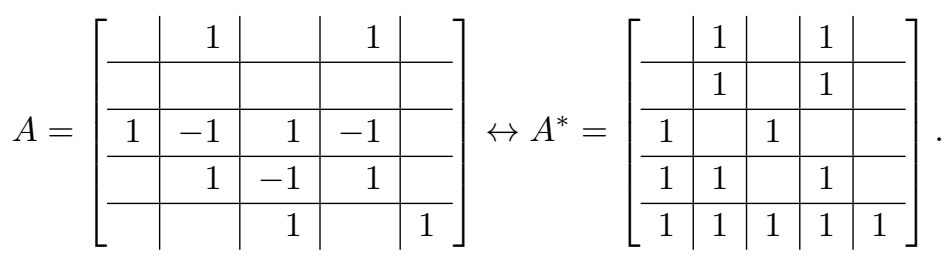

Let $A$ be an arbitrary $(0, \pm 1)$-matrix of size $m \times n$ with $\operatorname{PCSM} A^{*}=\left[a_{i j}^{*}\right] . \mathrm{A} 2 \times k$ matrix of the form

$$
\left[\begin{array}{llllll}
0 & 1 & 0 & \cdots & 1 & 0 \\
1 & 0 & 1 & \cdots & 0 & 1
\end{array}\right]
$$

will be called a zig-zag matrix. Note that $k$ must be odd, and the matrix has $(k-1) / 2$ ones in the first row, and $(k+1) / 2$ ones in the second row. We say that $A^{*}$ has the zig-zag property if for each $i<n$ the submatrix $A^{\prime}$ of $A^{*}$, obtained from rows $i$ and $i+1$ by deleting each column with two equal entries, is a zig-zag matrix.

We next characterize the partial-column-sums matrices of ASMs and of normalized SRMs. The characterization given for ASMs is equivalent to the well-known characterization of the corresponding MTs.

TheOREM 3.2. Let $A=\left[a_{i j}\right]$ be $a(0, \pm 1)$-matrix of size $m \times n$, and let $A^{*}=\left[a_{i j}^{*}\right]$ be its partial-columnsums matrix. Then

(i) $A$ is an $A S M$ if and only if $m=n, A^{*}$ is a $(0,1)$-matrix with $k$ ones in row $k(k \leq n)$, and $A^{*}$ has the zig-zag property.

(ii) $A$ is a normalized SRM if and only if $A^{*}$ is a $(0,1)$-matrix with only ones in the last row and

$$
\sum_{j=1}^{k} a_{i+1, j}^{*} \geq \sum_{j=1}^{k} a_{i j}^{*} \quad(k \leq n, i<m) .
$$

Proof. If $A$ is an SRM, then each column is alternating, so that $A^{*}$ is a $(0,1)$-matrix, and, if $A$ is also normalized, then the last row in $A^{*}$ is all ones.

(i) Assume that $A$ is an ASM. Then $m=n$, and $A$ has $i$ ones in row $i(i \leq n)$. Let $i<n$ and consider row $i+1$ of $A$. Since this row is alternating, by deleting its zeros we obtain

$$
\left[\begin{array}{llllll}
1 & -1 & 1 & -1 & \cdots & 1
\end{array}\right]
$$


Note that for each $j$ with $a_{i+1, j}=0$, we have $a_{i j}^{*}=a_{i+1, j}^{*}$. Moreover, if $a_{i+1, j}=1$, then $a_{i j}^{*}=0$ and $a_{i+1, j}^{*}=1$. Similarly, if $a_{i+1, j}=-1$, then $a_{i j}^{*}=1$ and $a_{i+1, j}^{*}=0$. This means that rows $i$ and $i+1$ satisfy the zig-zag property. Conversely, if the zig-zag property holds, then all the just mentioned properties of $A$ and $A^{*}$ in rows $i$ and $i+1$ hold, which means that row $i+1$ is alternating. Note that the first rows of $A$ and $A^{*}$ coincide and equal a $(0,1)$-vector with exactly one 1 .

(ii) Assume first that $A$ is a normalized SRM. It remains to prove (3.5) holds. Let $i<m$. Then

$$
\sum_{j=1}^{k} a_{i+1, j}^{*}-\sum_{j=1}^{k} a_{i, j}^{*}=\sum_{j=1}^{k}\left(a_{i+1, j}^{*}-a_{i, j}^{*}\right)=\sum_{j=1}^{k} a_{i+1, j} \geq 0 \quad(k \leq n),
$$

by the SRM property in row $i+1$. Conversely, assume $A^{*}$ is a $(0,1)$-matrix with only ones in the last row and that (3.5) holds. Then each column is alternating and has sum 1. Also, by (3.6), the partial row sums are nonnegative in each row $i \geq 2$. The same holds in the first row because this row coincides in $A$ and $A^{*}$, and $A^{*}$ is a $(0,1)$-matrix. So, $A$ is a normalized SRM.

Let $A^{(k)}$ denote the submatrix of $A$ consisting of its $k$ first columns $(k \leq n)$. Then, condition (3.5) for SRMs is equivalent to saying that $A^{(k)}$ has weakly increasing row sums $(k \leq n)$.

For an ASM $A$, the zig-zag property of the previous theorem may be expressed differently. For $i \leq m$ and $k \leq r_{i}\left(A^{*}\right)$, let $\xi_{k}\left(A^{*} ; i\right)$ denote the column of $A^{*}$ containing the $k^{\prime}$ th 1 in row $i$. It follows from part (i) of Theorem 3.2 that

$$
\xi_{k}\left(A^{*} ; i+1\right) \leq \xi_{k}\left(A^{*} ; i\right) \leq \xi_{k+1}\left(A^{*} ; i+1\right) \quad(k \leq i, i<n),
$$

implying the known monotonicity properties of MTs of ASMs, namely that the rising and falling diagonals are weakly increasing (that the rows are strictly increasing is trivial).

We next turn to a characterization of PMTs of matrices $A=\left[a_{i j}\right] \in \mathcal{S}_{m, n}^{++}$; thus, $A$ is a $(0,1)$-matrix with exactly one 1 in every column. First note that $A$ may be uniquely reconstructed from its PMT by letting, for each $j \leq n, a_{i j}=1$ for the row $i$ in which the topmost $j$ occurs; all the other entries in column $j$ are set equal to 0 .

Let $m, n \geq 1$ and $1 \leq i_{1}, i_{2}, \ldots, i_{n} \leq m$ be $n$ integers. Define an $m \times n$ matrix $B=\left[b_{i j}\right]$ recursively as follows:

1. Initially, let $B=O$.

2. for $j=1,2, \ldots, n$,

for $i=i_{j}, i_{j}+1, \ldots, m$,

let $k$ be the column of the leftmost 0 in row $i$ of $B$ and define $b_{i k}=j$.

The resulting matrix $B$ will also be denoted as $B^{i_{1}, \ldots, i_{n}}$. Then $b_{i j} \in\{0,1, \ldots, n\}(i \leq m, j \leq n)$ and each row consists of strictly increasing positive entries followed by zeros. We call $B$ a skew-interval matrix since once an integer occurs in a row, it occurs in each of the following rows in a left-to-right pattern. For instance

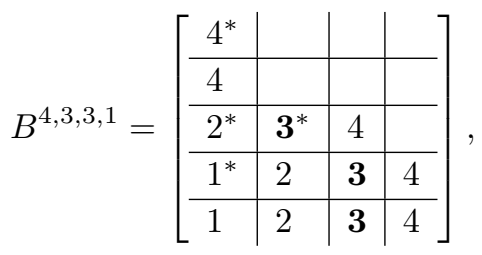


Electronic Journal of Linear Algebra, ISSN 1081-3810

A publication of the International Linear Algebra Society

Volume 37, pp. 613-639, September 2021.

where the entries in boldface indicate the skew-interval for $j=3$, and the entries with a $*$ are the topmost in each interval (and zeros are not shown).

LEMmA 3.3. Let $B$ be an $m \times n$ matrix. Then, $B$ is the PMT of some matrix in $\mathcal{S}_{m, n}^{++}$if and only if $B$ is a skew-interval matrix.

Proof. It is easy to check that: (i) if $A \in \mathcal{S}_{m, n}^{++}$has a 1 in position $\left(i_{j}, j\right)(j \leq n)$, then the PMT of $A$ is $B^{i_{1}, \ldots, i_{n}}$, and, conversely, (ii) a skew-interval matrix $B^{i_{1}, \ldots, i_{n}}$ is the PMT of the matrix $A \in \mathcal{S}_{m, n}^{++}$with a 1 in position $\left(i_{j}, j\right)(j \leq n)$.

For instance, the matrix $A$ reconstructed from the matrix $B^{4,3,3,1}$ above is

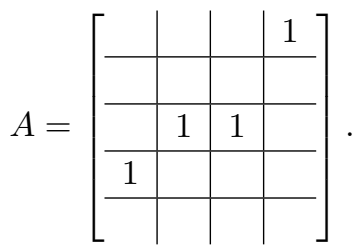

We remark that Lemma 3.3 gives a characterization of PMTs of matrices in $\mathcal{S}_{m, n}^{++}$, although it is not given in terms of simple inequalities between its entries. Still, it can be checked in polynomial time if a given integral matrix $B$ with entries in $\{0,1, \ldots, n\}$ is a skew-interval matrix. First, check that the last row is $(1,2, \ldots, n)$, and then, for $j=1,2, \ldots, n$, check that the entries equal to $j$ determine a skew-interval (as required in the definition).

Next, consider a general SRM $A \in \mathcal{S}_{m, n}$. The PMT of $A$ is monotone as described before (strictly increasing in rows etc.). The situation is then almost as above for $\mathcal{S}_{m, n}^{+}$, except that the skew-intervals for each integer is broken into subintervals (due to -1 's in $A$ ). There is additional structure (apart from monotonicity) on the PMT due to monotone partial row sums and this can be expressed in a more technical manner. Moreover, based on a given monotone PMT, we can use reconstruction as in the algorithm above, and find the unique underlying $A$. Then we can check if $A$ is an SRM.

We know that there is a bijection between ASMs and monotone triangles (of the same size $n$ ). It is natural to ask for a characterization of the MTs of permutation matrices in the above terms; the next corollary provides this.

Corollary 3.4. A monotone triangle $L$ of an ASM is the MT of a permutation matrix if and only if $L$ is skew-interval matrix.

Proof. The monotone triangle $L$ is the MT of a unique ASM $A$, so $L=L(A)$. Since $A=\left[a_{i j}\right]$ is an ASM, $A$ is a permutation matrix if and only if it has no negative entries. Moreover, there is a negative entry in position $(i, k)$ if and only if $A^{*}=\left[a_{p q}^{*}\right]$ contains, in column $k$, at least one 1 above row $i$ and at least one 1 below $i$, and $a_{i k}^{*}=0$ (by the ASM property of $A$ ). This, again, is equivalent to $L$ having the skew-interval property.

In Corollary 3.4 we start with the set of MTs of an ASM and characterize the set of MTs of permutation matrices within this set. In the next corollary, we start with the set of PMTs of SRMs and characterize the set of PMTs of matrices in $\mathcal{S}_{m, n}^{++}$within this set. The proof is similar.

Corollary 3.5. A PMT L of an $S R M$ is the PMT of a matrix in $\mathcal{S}_{m, n}^{++}$if and only if $L$ has the skew-interval property. 
A normalized SRM has all column sums equal to 1 but may have zero rows; the last row does not contain any -1 's. Let $\widehat{\mathcal{S}}_{m, n}$ denote the subset of $\mathcal{S}_{m, n}$ consisting of those matrices without any zero rows.

THEOREM 3.6. Given a positive integer $n$, the maximum integer $m$ such that $\widehat{\mathcal{S}}_{m, n} \neq \emptyset$ equals $\left(\begin{array}{c}n+1 \\ 2\end{array}\right)$.

Proof. Let $A$ be a matrix in $\widehat{\mathcal{S}}_{m, n}$. Since the column sums of $A$ all equal 1 and the row sum vector of $A$ is nonnegative, the sum of all the entries of $A$ equals $n$ and the row sum vector of $A$ contains at least $\max \{m-n, 0\} 0$ 's. The facts that the first nonzero in each row is a 1 and that the column sums equal 1 implies that the maximum number of nonzeros in columns $1,2,3, \ldots, n$ is, respectively, $1,3,5, \ldots, 2 n-1$. Hence $A$ contains at most $1+3+5+\cdots+(2 n-1)=n^{2}$ nonzeros. Let $\alpha$ be the set of rows with sum 0 , and let $\beta$ be the remaining rows so that $|\beta| \leq n$. Each row in $\alpha$ contains the same number of 1 's and -1 's, with at least one -1 and at least one 1 and so at least two nonzeros. Each row in $\beta$ contains at least one 1 . Thus $A$ contains at least $2|\alpha|+|\beta|$ nonzeros and so $2|\alpha|+|\beta| \leq n^{2}$. Summing this inequality and $|\beta| \leq n$ gives $2|\alpha|+2|\beta| \leq n^{2}+n$ so

$$
|\alpha|+|\beta| \leq \frac{n^{2}+n}{2}=\left(\begin{array}{c}
n+1 \\
2
\end{array}\right)
$$

Thus $m \leq\left(\begin{array}{c}n+1 \\ 2\end{array}\right)$

Equality can always be attained as we illustrate below for $n=5$ and $m=15$ :

$$
\left[\begin{array}{c|c|c|c|c} 
& & & & 1 \\
\hline & & & 1 & -1 \\
\hline & & 1 & -1 & \\
\hline & 1 & -1 & & \\
\hline 1 & -1 & & & \\
\hline & & & & 1 \\
\hline & & & 1 & -1 \\
\hline & & 1 & -1 & \\
\hline & 1 & -1 & & \\
\hline & & & & 1 \\
\hline & & & 1 & -1 \\
\hline & & 1 & -1 & \\
\hline & & & & 1 \\
\hline & & & 1 & -1 \\
\hline & & & & 1
\end{array}\right] .
$$

4. Related polytopes. It is proved in [10] that an $n \times n \mathrm{ASM}$ is the \pm 1 sum of at most $2 n-1$ permutation matrices. This implies that the partial-column-sums matrix (respectively, monotone triangle) of an ASM is a \pm 1 linear combination of the partial-column-sums matrices (respectively, monotone triangles) of at most $2 n-1$ permutation matrices.

Consider the partial-columns-sums matrix $C_{\sigma}$ corresponding to a permutation $\sigma=\left(i_{1}, i_{2}, \ldots, i_{n}\right)$. The column sum vector of $C_{\sigma}$ is the permutation

$$
\hat{\sigma}=(n+1, n+1, \ldots, n+1)-\left(j_{1}, j_{2}, \ldots, j_{n}\right),
$$


where $\sigma^{-1}=\left(j_{1}, j_{2}, \ldots, j_{n}\right)$. For example, let $n=8$ and $\sigma=(3,6,4,1,7,8,2,5)$ where $\sigma^{-1}=(4,7,1,3,8,2$, $5,6)$. Then

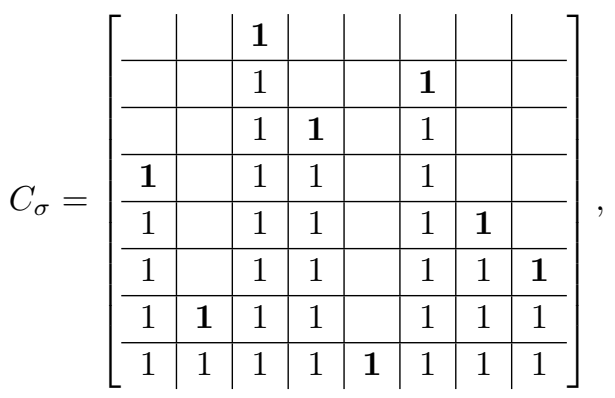

with column sum vector equal to

$$
(5,2,8,6,1,7,4,3)=(9,9,9,9,9,9,9,9)-(4,7,1,3,8,2,5,6) .
$$

Now instead of considering the convex hull of monotone triangles, we can consider the equivalent (since the partial-column-sums matrix determines the corresponding monotone triangle) convex hull of the partialcolumn-sums matrices for both permutation matrices and ASMs. As mentioned, since an ASM is a \pm 1 linear combination of permutation matrices, the partial-column-sums matrices of ASMs are \pm 1 linear combinations of partial-column-sums matrices of permutation matrices.

We study polytopes related to these matrix classes. Recall the linear map $S_{m, n}$ defined in (3.3), and we abbreviate $S_{n, n}$ to $S_{n}$.

For a matrix class $\mathcal{H} \subseteq M_{n}$ define

$$
S_{n}(\mathcal{H})=\left\{S_{n}(H): H \in \mathcal{H}\right\} .
$$

If $\mathcal{H}$ is a polytope, so is $S_{n}(\mathcal{H})$. We now consider this construction for the ASM polytope $\Gamma_{n}$ and its subpolytope $\Omega_{n}$ of $n \times n$ doubly stochastic matrices (the Birkhoff polytope). The advantage of considering $S_{n}\left(\Gamma_{n}\right)$ instead of $\Gamma_{n}$ is that $S_{n}\left(\Gamma_{n}\right)$ consists of nonnegative matrices while matrices in $\Gamma_{n}$, may have negative entries.

TheOrem 4.1. (i) $S_{n}\left(\Omega_{n}\right)$ equals the set of matrices $B=\left[b_{i j}\right] \in M_{n}$ satisfying

$$
\begin{array}{cl}
b_{i j} \geq b_{i-1, j} & (i, j \leq n), \\
\sum_{j=1}^{n}\left(b_{i j}-b_{i-1, j}\right)=1 & (i \leq n), \\
b_{n j}=1 & (j \leq n),
\end{array}
$$

where we define $b_{0 j}=0(j \leq n)$.

(ii) $S_{n}\left(\Gamma_{n}\right)$ equals the set of matrices $B=\left[b_{i j}\right] \in M_{n}$ satisfying

$$
\begin{array}{cl}
0 \leq \sum_{j^{\prime}=1}^{j}\left(b_{i j^{\prime}}-b_{i-1, j^{\prime}}\right) \leq 1 & (i, j \leq n), \\
0 \leq b_{i j} \leq 1 & (i, j \leq n), \\
\sum_{j=1}^{n}\left(b_{i j}-b_{i-1, j}\right)=1 & (i \leq n), \\
b_{n j}=1 & (j \leq n) .
\end{array}
$$


Proof. (i) We have $B \in S_{n}\left(\Omega_{n}\right)$ if and only if $A=S_{n}^{-1}(B) \in \Omega_{n}$. Then the result is obtained by inserting the expression for $A$ in (3.4) into the linear inequalities/equations defining $\Omega_{n}$; namely nonnegativity and that each line sum is 1 . (ii) This is obtained similarly, using the partial sum inequalities defining the ASM polytope.

Since the extreme points of $\Gamma_{n}$ are the $n \times n$ ASMs, the extreme points of $S_{n}\left(\Gamma_{n}\right)$ are the partial-columnsums matrices of the $n \times n$ ASMs and thus are $(0,1)$-matrices.

We next consider a polytope associated with monotone triangles (MTs) viewed, as we have previously, as Ferrers diagrams. An MT consists of positive integers associated with each position in the set $V=\{(i, j)$ : $1 \leq j \leq i, 1 \leq i \leq n\}$. Let $D=(V, E)$ be the digraph where, in view of the monotonicity properties of MTs, the edges indicate (i) successors in rows, (ii) successors in falling diagonals, and (iii) successors upward in columns. Thus, $E$ consists of the directed edges

(i) $((i, j),(i, j+1))$ for $j=1,2, \ldots, i-1$ and $i=1,2, \ldots, n$,

(ii) $((i+k-1, k),(i+k, k+1))$ for $k=1,2 \ldots, n-i$ and $i=1,2, \ldots, n$,

(iii) $((n+k-1, i+k-1),(n-k-2, i+k-1))$ for $k=1,2 \ldots, n-i$ and $i=1,2, \ldots, n$.

The digraph $D$ is clearly acyclic. The set $E_{h}$ of edges in (i) consists of the horizontal edges of $D$. The set $E_{d}$ of edges in (ii) consists of the diagonal edges, and the set $E_{v}$ of edges in (iii) consists of the vertical edges.

Let $\mathcal{M}_{n}$ denote the convex hull of all monotone triangles of size $n$. This is a polytope in the vector space $\mathcal{T}_{n}$ of all triangles of size $n$, i.e., functions $T: V \rightarrow \mathbb{R}$. A triangle in $\mathcal{T}_{n}$ will be denoted by $T=\left[t_{v}\right]$ where the subscript runs through $V$.

TheOrem 4.2. $\mathcal{M}_{n}$ equals the set of triangles $T=\left[t_{v}\right]$ satisfying

$$
\begin{array}{ll}
t_{v} \geq 0 & (v \in V), \\
t_{v} \geq t_{u}+1 & \left((u, v) \in E_{h}\right), \\
t_{v} \geq t_{u} & \left((u, v) \in E_{d}\right), \\
t_{v} \geq t_{u} & \left((u, v) \in E_{v}\right), \\
t_{n i}=i & (i=1,2, \ldots, n) .
\end{array}
$$

Proof. Let $P \subseteq \mathcal{T}_{n}$ be the polyhedron consisting of all real triangles $T=\left[t_{v}\right]$ satisfying the linear system in (4.11). This polyhedron is bounded as $0 \leq t_{v} \leq n$ for each $v \in V$ when $T=\left[t_{v}\right]$ satisfies (4.11). Therefore, $P$ is a polytope, i.e., the convex hull of a finite set of points.

Consider the linear system (4.11), and choose some ordering of the variables $t_{v}(v \in V)$, and let $t$ denote the corresponding vector. Then the subsystem consisting of the second, third, and fourth inequalities (those that have two variables) may be written as $A t \geq b$ where $A$ is a matrix whose rows are indexed by the edges in $E=E_{h} \cup E_{d} \cup E_{v}$, and the columns are indexed by the vertex set $V$. Every row in $A$ has two nonzeros, a 1 and $\mathrm{a}-1$. The vector $b$ contains the right-hand sides in the inequalities, so each component is 0 or 1 . The properties of the matrix $A$ means that it is the transpose of the vertex-edge incidence matrix of the digraph $D$ defined above. Therefore, $A$ is totally unimodular (see [16]) and, as all right-hand sides are integral, by general theory, the polytope $P$ is integral. This means that each of its vertices (extreme points) has only integral components. 
Electronic Journal of Linear Algebra, ISSN 1081-3810

A publication of the International Linear Algebra Society

Volume 37, pp. 613-639, September 2021.

Consider an integral vector $T=\left[t_{v}\right]$ satisfying (4.11). Then $T$ is nonnegative, strictly increasing in each row, and weakly increasing in each rising and falling diagonal. Moreover, the last row is $(1,2, \ldots, n)$. Thus, all integral vectors in the polyhedron $P$ are MTs. Since we just showed that all vertices of $P$ are integral, it follows that $P=\mathcal{M}_{n}$, as desired.

Note that the proof actually reveals more on the polytope $\mathcal{M}_{n}$. The matrix $A$ in the proof is the transpose of the incidence matrix of the digraph $D$. By network flow theory, it follows that all vertices of $\mathcal{M}_{n}$ correspond to spanning trees of the corresponding undirected graph. There is a one-to-one correspondence between the $n \times n$ ASMs $\mathcal{A}_{n}$ and the set of the MTs of order $n$. Thus, the convex hull of $\mathcal{A}_{n}$ corresponds to the convex hull $\mathcal{M}_{n}$ of the MTs of order $n$, and Theorem 4.2 describes $\mathcal{M}_{n}$. Since the set $\mathcal{A}_{n}$ of $n \times n$ alternating sign matrices is the set of extreme points of their convex hull, the set of extreme points of $\mathcal{M}_{n}$ is the set of MTs of ASMs of order $n$.

We refer to $[9,11,18]$ for more on polytopes related to ASMs and SRMs.

5. Expansions and deflations of SRMs and ASMs. Given an $m \times n$ normalized SRM $A$, we consider the possibility of an expansion of $A$ to another SRM $A^{\prime}$ of the same size (possibly an ASM if $m=n$ ) by changing one or more 0's to \pm 1 . If $A$ is already an $n \times n$ ASM, the result could be an ASM with a larger number of \pm 1 's; but it may also be a SRM that is not an ASM. For example, the ASM $I_{3}$ can be expanded to an SRM as shown below:

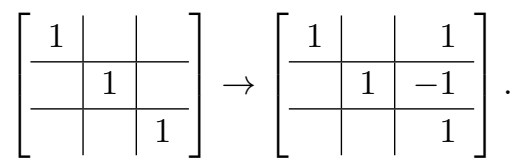

If $A$ is an SRM but not an ASM, we consider in addition the possibility of inserting new rows and columns anywhere throughout the matrix so that the resulting $t \times t \mathrm{ASM} A^{\prime}$ has $A$ as a submatrix. The minimum such $t$ we call the expansion parameter $\epsilon(A)$ of the $\operatorname{SRM} A$. If $A$ is an $n \times n$ ASM, we also consider the possibility of changing certain \pm 1 's to 0's resulting in an ASM with fewer \pm 1 's, a deflation of $A$ to another ASM.

ExAmple 5.1. The $1 \times 3 \mathrm{SRM} A$ of all 1 's can be expanded to the $5 \times 5$ diamond ASM as shown,

$$
A=\left[\begin{array}{lll}
1 & 1 & 1
\end{array}\right] \rightarrow\left[\begin{array}{r|r|r|r|r} 
& & 1 & & \\
\hline & 1 & -1 & 1 & \\
\hline 1 & -1 & 1 & -1 & 1 \\
\hline & 1 & -1 & 1 & \\
\hline & & 1 & &
\end{array}\right]
$$

by inserting two new columns and four new rows. It's expansion parameter $\epsilon(A)$ is clearly 5 . The $1 \times 5 \mathrm{SRM}$

$$
A_{1}=\left[\begin{array}{lllll}
1 & 0 & 1 & 0 & 1
\end{array}\right]
$$

can also be expanded to the same ASM by including four new rows and changing its two 0's to -1's. We also have $\epsilon\left(A_{1}\right)=5$. The $\mathrm{SRM} A$ in $(3.8)$ can be expanded to the $15 \times 15$ ASM $A^{\prime}$ by inserting 10 new columns as shown below: 


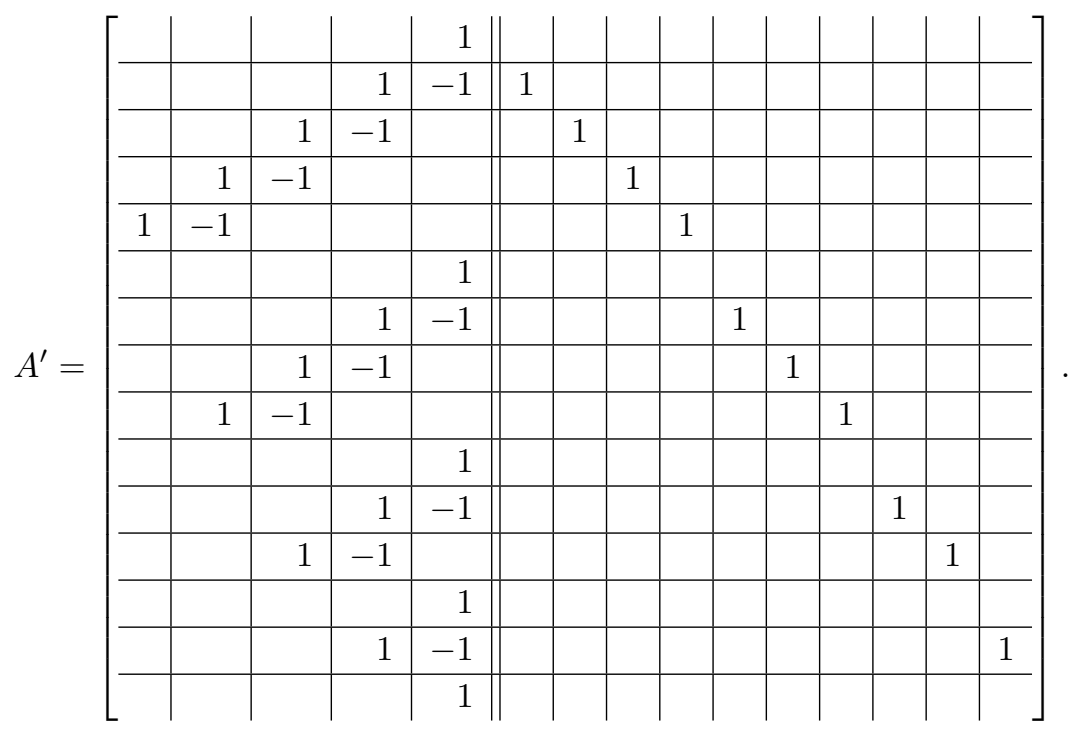

Its expansion parameter equals 15 since ten more columns are required to have a square matrix.

EXAmple 5.2. Consider the two $5 \times 5$ ASMs

$$
A^{\prime}=\left[\begin{array}{r|r|r|r|r} 
& & 1 & & \\
\hline & 1 & -1 & 1 & \\
\hline 1 & -1 & 1 & -1 & 1 \\
\hline & 1 & -1 & 1 & \\
\hline & & 1 & &
\end{array}\right] \text { and } A=\left[\begin{array}{r|r|r|r|r} 
& & 1 & & \\
\hline & 0 & 0 & 1 & \\
\hline 1 & 0 & 0 & -1 & 1 \\
\hline & 1 & -1 & 1 & \\
\hline & & 1 & &
\end{array}\right] \text {. }
$$

Then $A^{\prime}$ is an expansion of $A$ obtained by replacing the four specified 0's of $A$ as shown with \pm 1 's. Likewise $A$ is a deflation of $A^{\prime}$.

Example 5.2 motivates consideration of the following properties. An ASM $A$ is maximal ${ }^{1}$ provided that it is not possible to change a nonempty set of its 0's to \pm 1 's and obtain another ASM. The ASM $A^{\prime}$ in Example 5.2 is maximal, indeed, every diamond ASM (of which this is an example for $n=5$ ) is maximal as it contains the largest number of \pm 1 's for its size. The matrix $A$ is not maximal. In the other direction, an ASM is minimal provided that it is not possible to replace a nonempty set of \pm 1 's with 0 's to obtain another ASM. In Example 5.2, $A$ is minimal as is easily checked. Every permutation matrix is minimal.

An alternating cycle in an SRM is a cycle of rook-wise connected positions alternating between rows and columns whose entries also alternate between 1 and -1 . An alternating cycle in an SRM is a special alternating cycle provided its consecutive positions of 1's and -1 's in the same row, and similarly in the same column, have only 0's (possibly none) between them. In Example 5.2, $A$ is obtained from $A^{\prime}$ by replacing the \pm 1 's of a special alternating cycle of four positions with 0 's.

Let $A=\left[a_{i j}\right]$ be an $m \times n$ SRM. Define a graph $G_{A}^{*}=(V, E)$ associated with $A$ as follows. The vertex set consists of the set $V \subseteq\{(i, j): 1 \leq i \leq m, 1 \leq j \leq n\}$ of the positions of the matrix $A$ containing a 1 or -1 . Each edge is a pair of vertices (positions of \pm 1 's), in the same row or the same column, such that the two entries are 1 and -1 (in any order) without any \pm 1 's in between them. (If one omits the last condition

\footnotetext{
${ }^{1}$ Later we introduce a more precise terminology using partial orders.
} 
Electronic Journal of Linear Algebra, ISSN 1081-3810

A publication of the International Linear Algebra Society

Volume 37, pp. 613-639, September 2021.

for edges one obtains another graph $G_{A}$, which is of interest, although we do not consider it here.) The graph $G_{A}^{*}$ has some special properties. First, $G_{A}^{*}$ is bipartite with color classes $I$ and $J$ where $I$ consists of vertices $(i, j)$ containing a 1 and $J$ consists of the vertices $(i, j)$ containing a -1 . If $A$ is an ASM, each vertex in $I$ has degree equal to $0,1,2,3$, or 4 ; each vertex in $J$ has degree equal to 4 . An alternating cycle in $G_{A}^{*}$ is a cycle where consecutive vertices alternate between being in the same row or column. There is a one-to-one correspondence between the alternating cycles of $G_{A}^{*}$ and the special alternating cycles of $A$.

EXAMPLE 5.3. Let

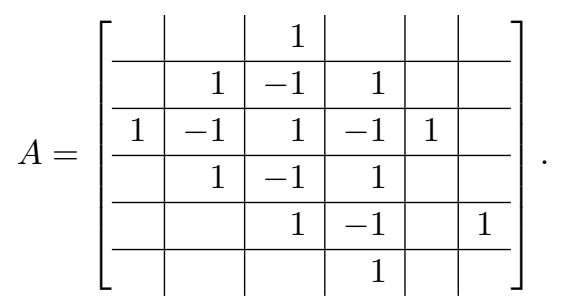

Then there are vertices of $G^{*}(A)$ corresponding to the 1's having each of the degrees $1,2,3$, and 4 .

The connected components $C_{1}, C_{2}, \ldots, C_{k}(k \geq 1)$ of $G^{*}(A)$ determine corresponding ASMs which may be interspersed within the rows and columns.

EXAMPLE 5.4. Let

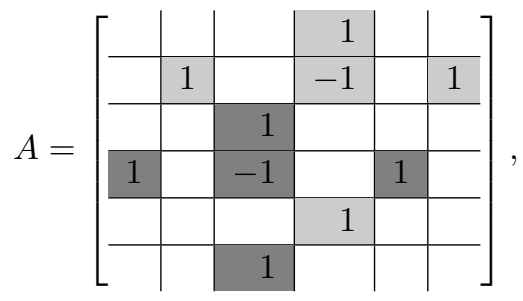

where the two connected components of $G^{*}(A)$ are given by the light and dark shadings.

LEMma 5.5. Let $A$ be an $n \times n$ ASM (resp. $m \times n$ SRM) and assume $A^{\prime}$ is obtained from $A$ by replacing all entries in a special alternating cycle in $A$ with zeros. Then $A^{\prime}$ is also an ASM (resp. SRM).

Proof. With the ASM assumption, this is clear as we delete from a row (respectively, a column) a 1 and a -1 with only 0's in-between: if a 1 from the special alternating cycle is followed by a -1 , then there is a first 1 after that -1 keeping the alternating property of rows and columns. If a -1 from the special alternating cycle is followed by a 1 , then there is a first 1 preceding the -1 keeping this alternating property.

Next, let $A=\left[a_{i j}\right]$ be an SRM. The previous argument shows that every column of $A^{\prime}$ is alternating. Next, consider a row in $A$. Then a similar argument for the sums in the previous paragraph can be used to show that the partial row sums in $A^{\prime}$ are nonnegative. Thus, $A^{\prime}$ is an SRM.

ExAmple 5.6. Consider the $7 \times 7$ diamond ASM, where we have shaded the positions of a special alternating cycle which is easily traced out (there are not any 0's between consecutive \pm 1 's): 


$\left[\begin{array}{r|r|r|r|r|r|r} & & & 1 & & & \\ \hline & & 1 & -1 & 1 & & \\ \hline & 1 & -1 & 1 & -1 & 1 & \\ \hline 1 & -1 & 1 & -1 & 1 & -1 & 1 \\ \hline & 1 & -1 & 1 & -1 & 1 & \\ \hline & & 1 & -1 & 1 & & \\ \hline & & & 1 & & & \end{array}\right]$.

Replacing its entries in the shaded positions with 0's, we obtain the ASM deflation given by

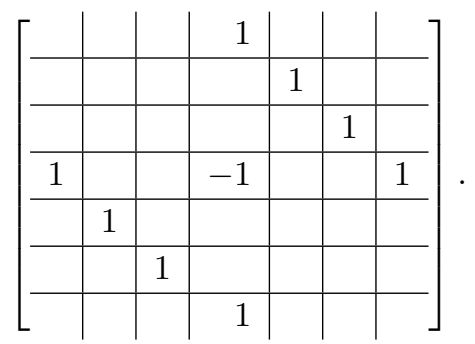

There is a well-known partial order on the set $\mathcal{A}_{n}$ of $n \times n$ ASMs, the Bruhat order $\preceq_{B}$. This partial order is the Dedekind-MacNeille (minimal lattice) completion of the partial order defined on the set $\mathcal{P}_{n}$ of $n \times n$ permutation matrices by transpositions. We have that for $A_{1}, A_{2} \in \mathcal{A}_{n}, A_{1} \preceq_{B} A_{2}$ if and only if $A_{1}$ can be obtained from $A_{2}$ by a sequence of transformations obtained by adding

$$
\left[\begin{array}{rr}
1 & -1 \\
-1 & 1
\end{array}\right]
$$

to $2 \times 2$ submatrices with all intermediate matrices contained in $\mathcal{A}_{n}$. Here we define two more natural partial orders on $\mathcal{A}_{n}$.

The pattern of a matrix $A$ is the set $\operatorname{Patt}(A)$ of positions of its nonzeros. If $A$ is an SRM or ASM, its pattern uniquely determines $A$, since the nonzeros in the columns of an SRM and ASM alternate between 1 and -1 starting with a 1 (the same property holds for the rows of ASMs but not necessarily for SRMs). Let $A_{1}$ and $A_{2}$ be two $n \times n$ ASMs. Then the pattern partial order $\preceq_{p}$ on $\mathcal{A}_{n}$ is defined by $A_{1} \preceq_{p} A_{2}$ provided that $\operatorname{Patt}\left(A_{1}\right) \subseteq \operatorname{Patt}\left(A_{2}\right)$. The pattern-plus partial order $\preceq_{p p}$ on $\mathcal{A}_{n}$ is $\operatorname{defined}$ by $A_{1} \preceq_{p p} A_{2}$ provided that every nonzero entry of $A_{1}$ equals the corresponding entry of $A_{2}$; thus the ASM $A_{2}$ is an expansion of the ASM $A_{1}$ and $A_{1}$ is a deflation of $A_{2}$ (possibly $\left.A_{1}=A_{2}\right)$. Note that if $A_{1} \preceq_{p p} A_{2}, \operatorname{then} \operatorname{Patt}\left(A_{1}\right) \subseteq \operatorname{Patt}\left(A_{2}\right)$ and hence $A_{1} \preceq_{p} A_{2}$. Thus, the pattern-plus partial order is a refinement of the pattern partial order. The subset $\mathcal{P}_{n}$ of $\mathcal{A}_{n}$ consisting of the $n \times n$ permutation matrices is a set of unrelated elements in both of these partial orders.

EXAMPLE 5.7. Let

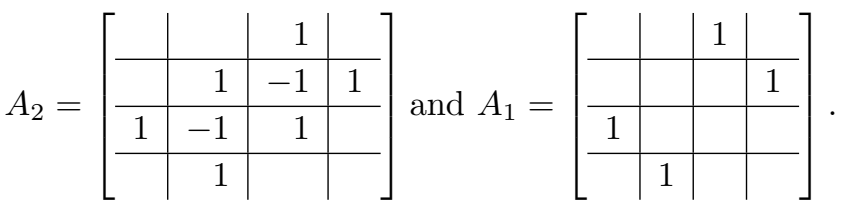

Then $A_{1} \preceq_{p} A_{2}$ and $A_{1} \preceq_{p p} A_{2}$. 
Electronic Journal of Linear Algebra, ISSN 1081-3810

EXAMPLE 5.8. Let

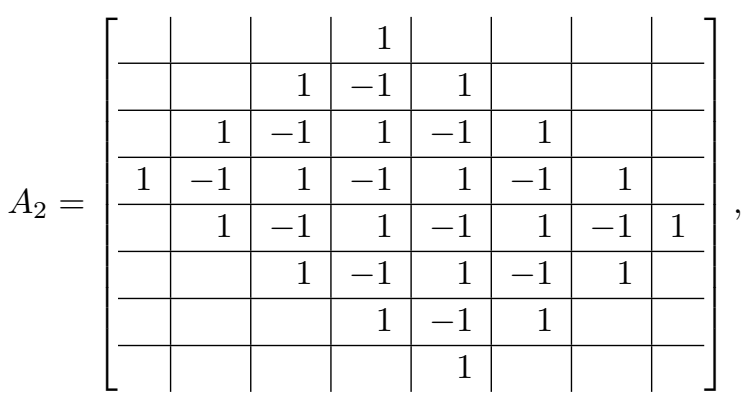

an $8 \times 8$ diamond ASM, and let

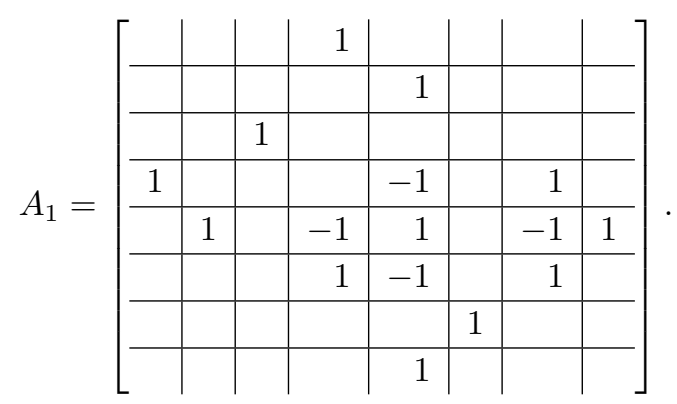

Then $A_{1}$ is an ASM with $\operatorname{Patt}\left(A_{1}\right) \subseteq \operatorname{Patt}\left(A_{2}\right)$ but $A_{1}$ does not agree with $A_{2}$ everywhere on $\operatorname{Patt}\left(A_{1}\right)$. Thus, $A_{1} \preceq_{p} A_{2}$ but $A_{1} \npreceq_{p p} A_{2}$. Thus, not surprisingly, the pattern-plus partial order is, in general, more restrictive than the pattern partial order.

As usual we write $A_{1} \prec_{p} A_{2}$ (resp. $\left.A_{1} \prec_{p p} A_{2}\right)$ if $A_{1} \preceq_{p} A_{2}$ (resp. $A_{1} \preceq_{p p} A_{2}$ ) and $A_{1} \neq A_{2}$. We determine the cover relation in the pattern-plus partial order which then determines uniquely the patternplus partial order. If a matrix $A_{1}$ is obtained from an ASM $A_{2}$ by replacing with 0's all the \pm 1 's of a special alternating cycle of $A_{2}$, then by Lemma 5.5, $A_{1}$ is an ASM and $A_{1} \prec_{p p} A_{2}$. We shall show that this property leads to a characterization of the cover relation of the pattern-plus partial order.

Lemma 5.9. Let $A_{1}$ and $A_{2}$ be $n \times n$ ASMs with $A_{1} \preceq_{p p} A_{2}$. Then $A_{2}-A_{1}$ is a $(0, \pm 1)$-matrix whose rows and columns sum to 0 and with the \pm 1 's in each row and column alternating. Moreover, $A_{2}-A_{1}=$ $C_{1}+C_{2}+\cdots+C_{k}$ where the $C_{i}$ are special alternating cycles with pairwise disjoint patterns.

Proof. Let $S$ be the set of positions in $A_{2}$ where the corresponding entry in $A_{2}$ is nonzero and the corresponding entry in $A_{1}$ is zero. For $i \leq n$, let $S_{i}$ be the intersection of $S$ with row $i$. Then, for some $k$, $S=S_{i_{1}} \cup S_{i_{2}} \cup \cdots \cup S_{i_{k}}$ where $1 \leq i_{1}<i_{2}<\cdots<i_{k} \leq n$ and each such $S_{i_{j}}$ consists of a positive even number of consecutive nonzero positions with zeros between. This follows from the fact that both $A_{1}$ and $A_{2}$ are ASMs. Thus, in the graph $G_{A_{2}}^{*}$, we get a number of pairs in $S$ where there is an edge between the first and the second position in $S$, an edge between the third and the fourth position etc. We call these edges horizontal. A similar property holds in every column, and the corresponding edges of $G_{A_{2}}^{*}$ are called vertical. This proves the first part of the lemma. Next, assuming $A_{1} \neq A_{2}$, choose a horizontal edge and for one of its end vertices choose an incident vertical edge. Then choose a horizontal edge incident to the previous vertex and continue like this until a special alternating cycle $C_{1}$ is formed. We now remove $C_{1}$ and repeat the overall argument for the new ASM and continue until eventually the horizontal and vertical edges are partitioned into special alternating cycles and $A_{1}$ is obtained. 
The following two examples give some insight in the present discussion.

ExAmple 5.10. Consider the ASM

$$
A=\left[\begin{array}{r|r|r|r|r|r} 
& & & & 1 & \\
\hline & & 1 & & & \\
\hline & 1 & -1 & 1 & -1 & 1 \\
\hline 1 & -1 & & & 1 & \\
\hline & & 1 & & & \\
\hline & 1 & & & &
\end{array}\right] .
$$

Then $A$ has an alternating cycle (as shaded) but it is not a special alternating cycle. In fact, it is easy to check (using the fact that there are six 1's in $A$ which are the only nonzeros in their row or column) that an ASM $B$ such that $B \preceq_{p} A$ implies $B=A$. Thus, $A$ is minimal in the pattern partial order and hence in the pattern-plus partial order. This example also shows that removing an alternating cycle which is not special may lead to a non-ASM. The ASM $A^{\prime}$ in Example 5.2 covers the ASM $A$ in the pattern partial order, but the ASMs in Example 5.6 do not satisfy a cover relation in the pattern partial order.

Example 5.11. Consider the ASM

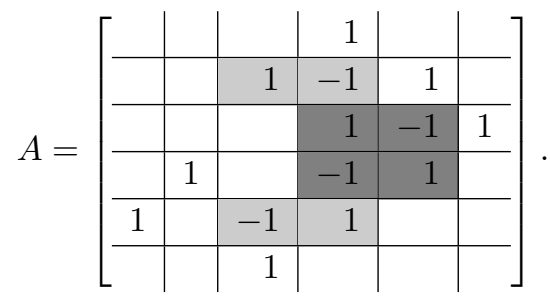

Then the variously shaded squares form a special alternating cycle and the darker squares form a special alternating cycle within it. Deleting this smaller alternating cycle, we obtain

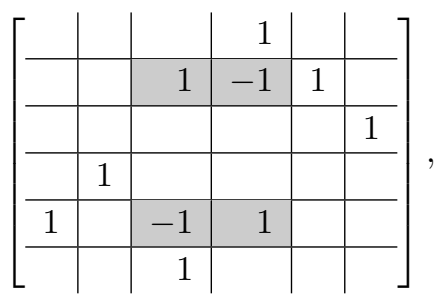

which now contains another special alternating cycle. Deleting it we obtain the permutation matrix

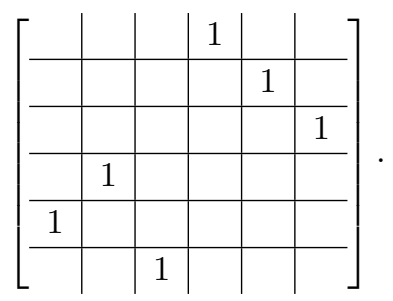

Note that the second special alternating cycle is initially not a special alternating cycle.

A minimal special alternating cycle is a special alternating cycle whose set of positions does not properly contain the set of positions of another special alternating cycle. By removing a special alternating cycle we mean replacing the \pm 1 's in its positions with 0 's. 
Electronic Journal of Linear Algebra, ISSN 1081-3810

A publication of the International Linear Algebra Society

Volume 37, pp. 613-639, September 2021.

TheOREM 5.12. Let $A_{1}$ and $A_{2}$ be two $n \times n$ ASMs. Then $A_{2}$ covers $A_{1}$ in the pattern-plus partial order if and only if $A_{1}$ can be obtained from $A_{2}$ by removing a minimal special alternating cycle.

Proof. First assume that $A_{1}$ is obtained from $A_{2}$ by removing a minimal special alternating cycle $C$ : $A_{2}-C=A_{1}$. Suppose that there is an ASM $B \neq A_{1}, A_{2}$ such that $B$ is an expansion of $A_{1}$ and a deflation of $A_{2}: A_{1} \prec B \prec A_{2}$. Then by Lemma $5.9 B-A_{1}$ contains a special alternating cycle $C^{\prime}$ which is a proper subset of the alternating cycle $C$, contradicting the minimality of $C$.

Now suppose that $A_{2}$ covers $A_{1}$ in the pattern-plus partial order. Then by Lemma 5.9, $A_{2}-A_{1}=$ $C_{1}+C_{2}+\cdots+C_{k}$ where $k \geq 1$ and the $C_{i}$ are special alternating cycles with pairwise disjoint patterns. If $k>1$, then clearly $A_{2}$ does not cover $A_{1}$. Hence, $k=1$ and the cycle $C_{1}$ must clearly be minimal, and the lemma follows.

We now briefly turn to the cover relation for the stricter pattern partial order $\preceq_{p}$. If $A_{1}$ and $A_{2}$ are two $n \times n$ ASMs, then since $A_{1} \preceq_{p p} A_{2}$ implies that $A_{1} \preceq_{p} A_{2}$, it follows that if $A_{2}$ covers $A_{1}$ in the pattern partial order $\preceq_{p}$ and $A_{1} \preceq_{p p} A_{2}$, then $A_{2}$ covers $A_{1}$ in the pattern-plus partial order $\preceq_{p p}$. However, the cover relation in the pattern partial order is more complicated than in the pattern-plus partial order (see the characterization in Theorem 5.12). The following example illustrates this complexity.

ExAmple 5.13. Consider the following two ASMs
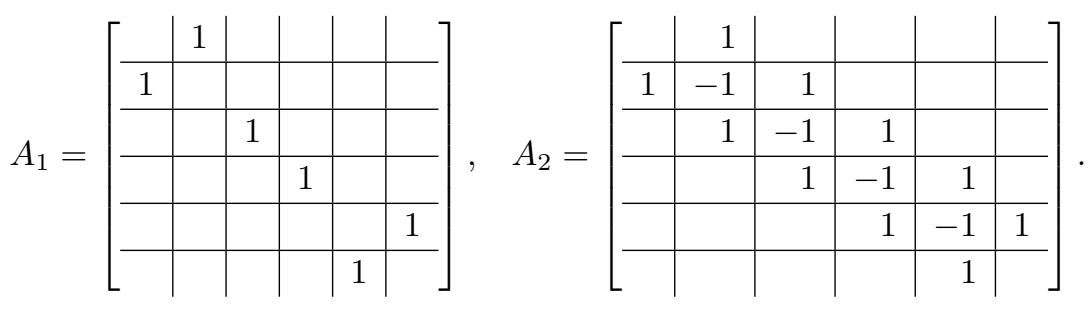

Then $A_{1} \prec_{p} A_{2}$ since $\operatorname{Patt}\left(A_{1}\right) \subset \operatorname{Patt}\left(A_{2}\right)$. Note that the entries in positions $(3,3)$ and $(4,4)$ in $A_{1}$ equal 1 while the corresponding entries in $A_{2}$ equal -1. Thus, $A_{1} \nprec_{p p} A_{2}$. We now prove that $A_{2}$ covers $A_{1}$ in the pattern partial order. Assume $A=\left[a_{i j}\right]$ is an ASM with $A_{1} \preceq_{p} A \preceq_{p} A_{2}$. Clearly, $a_{12}=a_{21}=a_{56}=a_{65}=1$. Consider first the case when $a_{22}=0$. Then $a_{2 i}=a_{i 2}=0$ for $i=3,4,5,6$, and therefore $a_{33}$ (which must be nonzero) equals 1 . This again implies $a_{3 i}=a_{i 3}=0$ for $i=4,5,6$. Then a similar argument gives $a_{44}=1$, and eventually that $A=A_{1}$. Next, consider the remaining case when $a_{22}$ is nonzero, so $a_{22}=-1$. Then $a_{23}=a_{32}=1$. Next, $a_{33}$ must be nonzero, and it equals -1 . This implies $a_{34}=a_{43}=1$. Similarly, $a_{44}$ must be nonzero, and therefore $a_{44}=-1$ and $a_{45}=a_{54}=1, a_{55}=-1$, so $A=A_{2}$. This shows that $A_{2}$ covers $A_{1}$ in the pattern partial order.

The previous example, and the argument for the cover property, can be extended to tridiagonal diamond ASMs of order $n$. Note that in Example 5.13 one may obtain $A_{1}$ from $A_{2}$ by adding three matrices corresponding to 4 -cycles in the bipartite graph of $A_{2}$.

We conclude this section with the following property of the pattern partial order.

Theorem 5.14. Let $A_{1}$ and $A_{2}$ be two $n \times n$ ASMs such that $A_{1} \prec_{p} A_{2}$ but $A_{1} \nprec_{p p} A_{2}$. Then

$$
\left|\operatorname{Patt}\left(A_{2}\right)\right|-\left|\operatorname{Patt}\left(A_{1}\right)\right| \geq 6
$$

Proof. Suppose that $A_{1}$ and $A_{2}$ differ in position $(i, j)$ where $A_{2}$ has a 1 and $A_{1}$ has a -1 . Since the \pm 1 's in each row and column of an ASM alternate and since $\operatorname{Patt}\left(A_{1}\right) \subseteq \operatorname{Patt}\left(A_{2}\right), A_{1}$ has at least one more 
Electronic Journal of Linear Algebra, ISSN 1081-3810

A publication of the International Linear Algebra Society

Volume 37, pp. 613-639, September 2021.

zero than $A_{2}$ in the positions in the partial columns above and below position $(i, j)$ and at least one more zero than $A_{2}$ in the positions in the rows to the left and the right of position $(i, j)$. Let $j_{1}$ and $j_{2}$ be the columns of the two last mentioned zeros. Then each of columns $j_{1}$ and $j_{2}$ contains at least one more position where $A_{1}$ contains a zero while $A_{2}$ contains a nonzero (since the number of nonzeros in a column an ASM is odd). So, $\left|\operatorname{Patt}\left(A_{2}\right)\right|-\left|\operatorname{Patt}\left(A_{1}\right)\right| \geq 6$. A similar argument works if $A_{2}$ has a -1 and $A_{1}$ has a 1 in position $(i, j)$.

6. Minimal and maximal elements. We study the minimal and maximal elements in the previously defined partial orders, and we start with some results for the pattern-plus partial order.

The following corollary follows immediately from Theorem 5.12 and Lemma 5.9.

Corollary 6.1. An $n \times n$ ASM is a minimal element in the pattern-plus partial order if and only if it does not contain a special alternating cycle, i.e., $G_{A}^{*}$ is acyclic.

Considering again Example 5.10, we note that the graph $G_{A}^{*}$ is acyclic and this proves that $A$ is minimal in the pattern-plus partial order. As a consequence of Corollary 6.1 one can check efficiently if a given ASM $A$ is minimal in the pattern partial order: this is done by breadth-first-search, which determines the connected components of $G_{A}^{*}$ and for each component decides if a cycle exists.

Let $A$ be an $n \times n$ ASM. We describe an expansion of $A$ into an $(n+2) \times(n+2)$ ASM $A^{\prime}$. First add as last columns two unit vectors $-e_{i}$ and $e_{i}$ for some $i \leq n$. Then add a row on top and one at the bottom, both unit vectors with a 1 in column $n+1$, the same column as the new -1 . The resulting matrix $A^{\prime}$ is also an ASM. A similar expansion exists by initially adding two new initial columns, or by similar operations for rows first. We call every such $A^{\prime}$ a 2-expansion of $A$. Thus, there are $4 n$ such 2 expansions of $A$. For instance, the diamond ASM of order 3 is a 2-expansion of the $1 \times 1$ permutation matrix.

Lemma 6.2. Let $A$ be an $n \times n$ ASM. Assume that $A$ is minimal in the pattern-plus partial order. Then every 2-expansion $A^{\prime}$ of $A$ is also minimal in the pattern-plus partial order.

Proof. The graph $G_{A^{\prime}}^{*}$ is obtained $G_{A}^{*}$ by adding vertices corresponding to the four new nonzeros, and four new edges, each incident to the position where the -1 was added. Thus, these edges constitute a 4 -star where one pendant vertex of the star is identified with a vertex in $G_{A}^{*}$. By Corollary $6.1 G_{A}^{*}$ is acyclic, and by these remarks, $G_{A^{\prime}}^{*}$ is also acyclic, so $A^{\prime}$ is minimal in the pattern-plus partial order.

From this it is clear how to construct large classes of pattern-plus minimal matrices: one starts with an pattern-plus minimal matrix, e.g., a permutation matrix of some order, and successively makes 2-expansions. All the constructed matrices are then minimal in the pattern-plus partial order.

Let $\tau(A)$ be the number of nonzeros in a matrix $A$, and let $\tau_{p}(n)$ be the maximum number of nonzeros in an $n \times n$ ASM that is minimal in the pattern partial order.

TheOrem 6.3. ( $i)$ For $n$ odd, $\tau_{p}(n)=2 n-1$. An ASM is minimal in the pattern-plus partial order and satisfies $\tau(A)=2 n-1$ if and only if $G_{A}^{*}$ is a (spanning) tree.

(ii) For $n$ even, $\tau_{p}(n)=2 n-2$.

Proof. Let $n \geq 1$, and let $A$ be an minimal ASM in the pattern-plus partial order. By Corollary 6.1, $G_{A}^{*}$ is acyclic. The horizontal edges in $G_{A}^{*}$ join two consecutive nonzero entries in a row, so the number of horizontal edges in a row is one less than its number of nonzeros. Therefore, the number of horizontal 
edges is $\tau(A)-n$. Similarly, the number of vertical edges in $G_{A}^{*}$ is $\tau(A)-n$, and the total number of edges is $2(\tau(A)-n)$. Note that $\tau(A)$ also equals the number of vertices of $G_{A}^{*}$. Since $G_{A}^{*}$ is acyclic, its number of edges is at most $\tau(A)-1$. This proves that $2(\tau(A)-n) \leq \tau(A)-1$. Therefore, $\tau(A) \leq 2 n-1$ and $\tau_{p}(n) \leq 2 n-1$. Moreover, it follows that $\tau(A)=2 n-1$ if and only if $G_{A}^{*}$ is a (spanning) tree.

Now, let $n$ be odd, say $n=2 k+1$. Let $A^{(n)}=\left[a_{i j}\right]$ be an ASM with row $k+1$ having no zeros (so its entries are $1,-1, \ldots, 1)$, and where the $j$ 'th column, for $j$ even, contains two ones, one in a row $<k+1$ and the other in a row $>k+1$. All these ones are placed in different rows, and all other entries are zero. Then $A^{(n)}$ has $\tau=2 n-1$ nonzeros and $G_{A^{(n)}}^{*}$ is a tree, so this matrix is minimal in the pattern-plus partial order, and $\tau(A)=2 n-1$, as desired. Below we show $A^{(5)}$

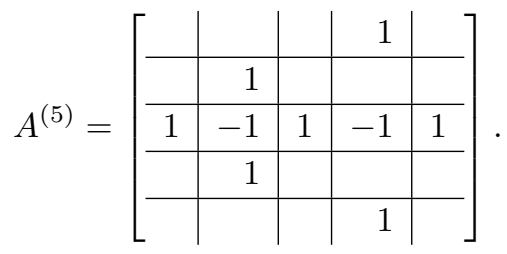

Next we consider $n$ even. Then $\tau_{p}(n) \leq 2 n-1$ as the proof above also works for $n$ even. Let $A$ be an ASM of size $n \times n$. Every line in $A$ has an odd number of nonzeros, and as $n$ is even, it follows that the number $\tau(A)$ of nonzeros in $A$ is an even number. But then $\tau(A)$ cannot be equal to the odd number $2 n-1$. This proves that $\tau_{p}(n) \leq 2 n-2$. Let $A$ be the direct sum $A=A^{(n-1)} \oplus J_{1}$ where $J_{1}$ is the $1 \times 1$ matrix with entry 1 . Then $\tau(A)=(2(n-1)-1)+1=2 n-2$, and (ii) follows.

THEOREM 6.4. The pattern partial order and pattern-plus partial order on the set $\mathcal{A}_{n}$ have the same set of minimal elements.

Proof. Clearly every minimal ASM in the pattern partial order is also minimal in the pattern-plus partial order. Now assume that $A$ is an $n \times n$ minimal ASM in the pattern-plus partial order and that $A^{\prime}$ is an $n \times n$ ASM with $\operatorname{Patt}\left(A^{\prime}\right) \subseteq \operatorname{Patt}(A)$. Let $S=\operatorname{Patt}(A) \backslash \operatorname{Patt}\left(A^{\prime}\right)$. We show that $S=\emptyset$, which means that $A$ and $A^{\prime}$ have the same pattern and therefore are equal. So, assume $S \neq \emptyset$. By the ASM property every line in $A$ contains an even number of elements in $S$. We choose an element $v_{1} \in S$ and another element $v_{2} \in S$ in the same row. Then choose $v_{3} \in S$ in the same column as $v_{2}$. Continuing like this, alternating with rows and columns, we obtain a cycle $C$ of positions in $A$. Note that the subgraph of $G_{A}^{*}$ induced by the nonzero positions in a row (or column) is a path, where edges connect consecutive nonzero positions. Each pair of consecutive vertices of the cycle $C$ is in the same row or column of $A$, so therefore they may be connected by some edges in the paths just mentioned for $G_{A}^{*}$. As a result, we obtain a cycle in $G_{A}^{*}$. Since $A$ is minimal in the pattern-plus partial order, $G_{A}^{*}$ is acyclic and so we have a contradiction. This shows that $S=\emptyset$, as desired.

While all permutation matrices are minimal in both the pattern and pattern-plus partial orders, they are not all maximal. For instance

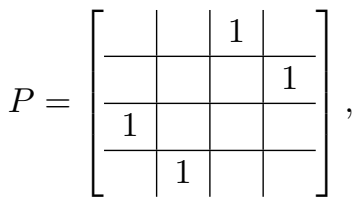


is not maximal in either of these partial orders as the ASM below shows:

$$
A=\left[\begin{array}{r|r|r|r} 
& & 1 & \\
\hline & 1 & -1 & 1 \\
\hline 1 & -1 & 1 & \\
\hline & 1 & &
\end{array}\right] .
$$

ExAmple 6.5. Consider the $n \times n$ identity matrix $I_{n}$ and its pattern $\operatorname{Patt}\left(I_{n}\right)$. Let $A$ be an $n \times n$ ASM with $\operatorname{Patt}\left(I_{n}\right) \subseteq \operatorname{Patt}(A)$. Then $A$ must have 1 's in both positions $(1,1)$ and $(n, n)$, and hence $A$ has 0 's in all other positions in rows and columns 1 and $n$. Crossing out rows and columns 1 and $n$ of $A$, we are left with an $\mathrm{ASM} A^{\prime}$ with $\operatorname{Patt}\left(I_{n-2}\right) \subseteq \operatorname{Patt}\left(A^{\prime}\right)$. Continuing like this we conclude that $A=I_{n}$. Thus, $I_{n}$ is a maximal element in the pattern partial order, and hence in the pattern-plus partial order.

We can generalize the construction in the previous example to get a class of permutation matrices that are maximal in these partial orders. In an $n \times n$ matrix let its corner positions be the positions $(1,1),(1, n)$, $(n, 1)$, and $(n, n)$. Let $P$ be a permutation matrix of order $n$ constructed as follows: (i) put a 1 in one of its corner positions and (ii) strike out the corresponding row and column and repeat the procedure for the remaining matrix of order $n-1$, etc. We call $P$ a corner permutation matrix. An example is

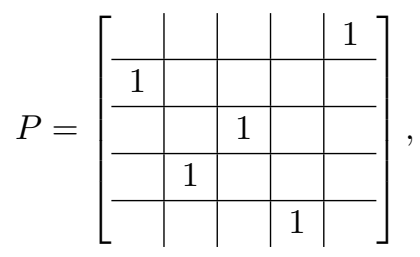

where we constructed $P$ by adding ones in the positions $(1,5),(5,4),(2,1),(3,3),(4,2)$; each being corner positions of the respective matrices.

LEMma 6.6. Let $P$ be a corner permutation matrix. Then $P$ is a maximal element in both the pattern and pattern-plus partial orders.

Proof. Let $A$ be an ASM with $P \preceq p A$. We prove that $A=P$, by induction on $n$. For $n=1$, this is trivial. Since $P$ is a corner permutation matrix it has a 1 in some corner position $(i, j)$, and then $A$ also has a 1 in the same position. This is due to the ASM property and the assumption $\operatorname{Patt}(P) \subseteq \operatorname{Patt}(A)$. Moreover, the other entries in row $i$ and column $j$ are zero, in both $P$ and $A$. Striking out row $i$ and column $j$, gives matrices $P^{\prime}$ and $A^{\prime}$ of order $n-1$, obtained from $P$ and $A$, respectively. Then $\operatorname{Patt}\left(P^{\prime}\right) \subseteq \operatorname{Patt}\left(A^{\prime}\right)$, so by induction $A^{\prime}=P^{\prime}$, and therefore $A=P$. So $P$ is maximal in both the pattern and pattern-plus partial orders.

Next we characterize the permutation matrices that are maximal in the pattern-plus partial order on $\mathcal{A}_{n}$.

THEOREM 6.7. Let $P$ be a permutation matrix of order $n$, and let the corresponding permutation be $\sigma=\left(i_{1}, i_{2}, \ldots, i_{n}\right)$. Then $P$ is maximal in the pattern-plus partial order in $\mathcal{A}_{n}$ if and only if there is no subsequence $i_{k_{1}}, i_{k_{2}}, i_{k_{3}}, i_{k_{4}}$ with the same relative relations as $3,4,1,2$, or as $2,1,4,3$, that is, $\sigma$ is 3412 avoiding and 2143-avoiding.

Proof. Assume that $P$ is maximal in the pattern-plus partial order. Then there cannot exist a subsequence $i_{k_{1}}, i_{k_{2}}, i_{k_{3}}, i_{k_{4}}$ with the same relative relations as $3,4,1,2$, or as $2,1,4,3$. This is because we can then add a special alternating cycle with four elements, as in the example above. 
To prove the converse, assume that $P$ is not maximal in the pattern-plus partial order. Then we can add a special alternating cycle $C$ to $P$ and obtain an ASM $A=\left[a_{i j}\right]$ with $P \prec_{p} A$. We say that an entry in $C$ with $a_{i j}=-1$ is NE (north-east) if its two adjacent positions in $C$ (both with a 1 ) are to the left in the same row, and below in the same column. Similarly we define the notions NW (north-west), SE (south-east), and SW (south-west); all these only refer to positions where $A$ has a -1 . Let $i_{1}$ be the first row in $A$ that contains an element in $C$, and let $\left(i_{1}, j_{1}\right)$ and $\left(i_{1}, j_{2}\right)$ be two consecutive elements in $C$ with $j_{1}<j_{2}$ (so they are adjacent in $\left.G_{P}^{*}\right)$; see the figure below.

\begin{tabular}{l|l|l|l|l|l|l|l|l|l} 
& & & & & & $j_{1}$ & & $j_{2}$ & $\ldots$ \\
\hline$i_{1}$ & & & & & & + & & - & \\
\hline & & & & & & & & & \\
\hline & & & & & & & & + & \\
\hline$i_{2}$ & & & & & + & - & & & \\
\hline & & & & + & - & & & & \\
\hline & & & & & & & & &
\end{tabular}

Assume $a_{i_{1} j_{2}}=-1$; the opposite case may be treated with similar arguments. Then $\left(i_{1}, j_{2}\right)$ is a NE position. Let $i_{2}$ be such that $\left(i_{2}, j_{1}\right)$ is adjacent to $\left(i_{1}, j_{1}\right)$ in $C$. So then $i_{2}>i_{1}, a_{i_{1} j_{1}}=1$ and $a_{i_{2} j_{1}}=-1$. Consider first the case when $\left(i_{2}, j_{1}\right)$ is a SW position. Then $P$ contains a 1 to the left of $\left(i_{2}, j_{1}\right)$ in the same row, and also a 1 below $\left(i_{2}, j_{1}\right)$ in the same column. Similarly, as $\left(i_{1}, j_{2}\right)$ is a NE position, $P$ contains a 1 to the right of $\left(i_{1}, j_{2}\right)$ in the same row, and also a 1 above $\left(i_{1}, j_{2}\right)$ in the same column. These four 1 's correspond to a subsequence of the permutation with the relative pattern $3,4,1,2$, as desired.

It remains to consider the case when $\left(i_{2}, j_{1}\right)$ is not a SW position. Then it must be a SE position. In order to return to column $j_{2}$ the cycle $C$ must contain a NW or SW position. If it contains a SW position, we are done because this position and position $\left(i_{1}, j_{2}\right)$ leads to a relative pattern $3,4,1,2$ as above. Alternatively, $C$ contains a NW position, but in that case this position must be "north-east" of an SE position (see figure), and this means that the permutation has a relative pattern $2,1,4,3$, which proves the theorem.

Now let $A$ be an SRM. Assume $G_{A}^{*}$ has $k$ vertex-disjoint cycles $C_{1}, C_{2}, \ldots, C_{k}$. Then the principal order ideal $\mathcal{I}_{A}$ of $A$ in the pattern-plus partial order, consisting of all SRMs $B$ with $B \preceq_{c} A$, contains at least $2^{k}$ SRMs. Indeed, by removing any subset of the corresponding alternating cycles one obtains $2^{k}$ distinct SRMs in $\mathcal{I}_{A}$.

In [7] it is shown that an $n \times n(0,-1)$-matrix of the form

$\left[\begin{array}{c||ccc||c}0 & 0 & \cdots & 0 & 0 \\ \hline \hline 0 & & & & 0 \\ \vdots & & -P_{n-2} & & \vdots \\ 0 & & & & 0 \\ \hline \hline 0 & 0 & \cdots & 0 & 0\end{array}\right]$,

where $P_{n-2}$ is an $(n-2) \times(n-2)$ permutation matrix, (such matrices are called bordered-permutation $(0,-1)$-matrices in [7]) can always be completed to an ASM by changing certain 0's (necessarily $2(n-1)$ of them) to 1's; moreover, the inductive proof shows (although not stated there) that the resulting ASM has $(n-2)$ rows and $(n-2)$ columns with 3 nonzero entries where the first and last rows and columns have exactly one 1 . 
EXAMPLE 6.8. Below is a bordered-permutation $(0,-1)$-matrix and two completions to an ASM:

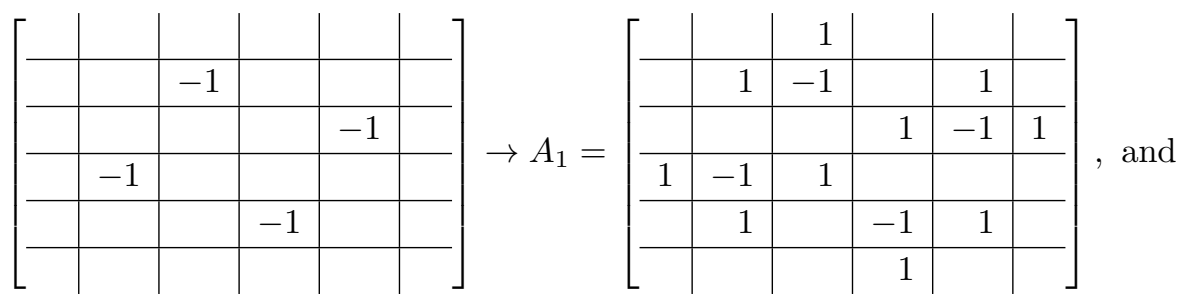

$$
A_{2}=\left[\begin{array}{r|r|r|r|r|r} 
& & 1 & & & \\
\hline & 1 & -1 & & 1 & \\
\hline & & 1 & & -1 & 1 \\
\hline 1 & -1 & & 1 & & \\
\hline & 1 & & -1 & 1 & \\
\hline & & & 1 & &
\end{array}\right] .
$$

Considering, e.g., $A_{1}$, we see that there are two permutation matrices $P \leq A_{1}$, namely
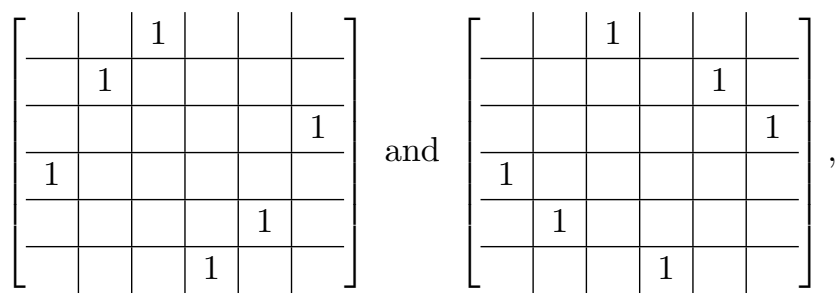

and both of these are covered by $A_{1}$ in the pattern and pattern-plus partial orders. In contrast,

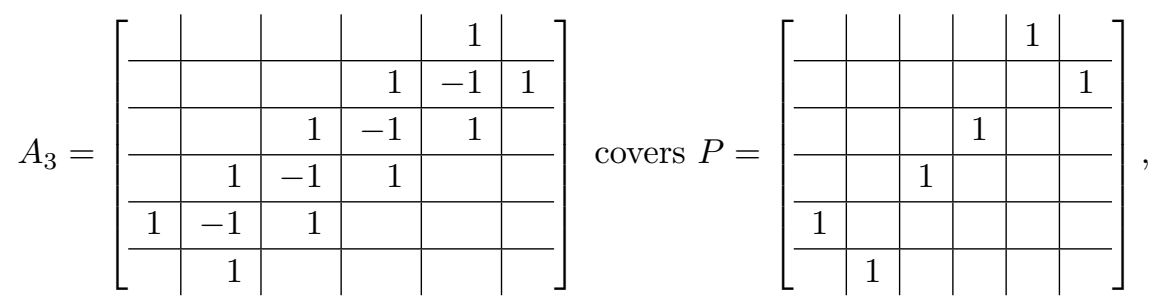

in the pattern partial order but not in the pattern-plus partial order. However, $P$ is covered by

$\left[\begin{array}{r|r|r|r|r|r} & & & & 1 & \\ \hline & & 1 & & -1 & 1 \\ \hline & 1 & -1 & 1 & & \\ \hline & & 1 & -1 & 1 & \\ \hline 1 & -1 & & 1 & & \\ \hline & 1 & & & & \end{array}\right]$,

in the pattern-plus partial order. 
Electronic Journal of Linear Algebra, ISSN 1081-3810

A publication of the International Linear Algebra Society

Volume 37, pp. 613-639, September 2021.

The previous example shows some ASMs with three nonzeros in every row and column, except the first and last. This is an interesting class of ASMs, and we now consider some properties of this class involving the pattern partial order. Consider the diamond ASM of order 2

$$
D_{2}=\left[\begin{array}{r|r|r} 
& 1 & \\
\hline 1 & -1 & 1 \\
\hline & 1 &
\end{array}\right]
$$

Its term rank is 2 , and there is no permutation matrix $P$ with $P \preceq_{p} A$. We show that this is the only matrix with this property among ASMs with three nonzeros in all lines expect the first and last row and column.

TheOREM 6.9. Let $n \geq 3$, and let $A$ be an $n \times n$ ASM with exactly three nonzeros in rows and columns $2,3, \ldots, n-1$. Then $A$ is a completion of a bordered-permutation $(0,-1)$-matrix, and there is a permutation matrix $P$ with $P \preceq_{p} A$ if and only if $n \geq 4$; moreover, this permutation matrix $P$ contains exactly $(n-4)$ -1 's and these occur in rows and columns $2,3, \ldots, n-1$.

Proof. First, if $n=3$, the only candidate is the diamond $D_{2}$ and, as remarked above, there is no permutation matrix $P$ with $P \preceq_{p} A$. So, assume $n \geq 4$. We shall prove that $A$ has term rank $n$.

Claim 1: The ones in the first and last rows are in different columns. Also the ones in the first and last columns are in different rows.

Proof of Claim 1: Assume that $A=\left[a_{i j}\right]$ satisfies $a_{1 j}=a_{n j}=1$ for some $j$ (so then $2 \leq j \leq n-1$ ). The only possible position for the -1 in row 2 is in column $j$ (otherwise there would be another 1 in the first row), so $a_{2 j}=-1$. For the same reason, by considering row $n-1$, we obtain $a_{n-1, j}=-1$. But then column $j$ has two -1 's, a contradiction. Therefore, the ones in the first and last rows must be in different columns. Similarly, the ones in the first and last columns must be in different rows. This proves Claim 1.

Let $I$ be a set of rows in $A=\left[a_{i j}\right]$, and define $J_{I}$ as the set of those columns $j$ for which $a_{i j}$ is nonzero for some $i \in I$.

Claim 2: $\left|J_{I}\right| \geq|I|$.

Proof of Claim 2: Consider first the case when $I$ is nonempty and does not contain the first or last row. Then each row in $I$ contains a -1 and these -1 's are in different columns (as there are three nonzeros in such columns). Thus, $A$ is a completion of a bordered-permutation $(0,-1)$-matrix. Moreover, the first and last 1 in these rows are in two different columns from where the -1 's are. Therefore $\left|J_{I}\right| \geq|I|+2$. This inequality implies that $\left|J_{I}\right| \geq|I|$ for any set $I$ of rows as long as $I$ contains at least one of the rows $2,3, \ldots, n-1$. If $I$ contains only the first or last row, then $\left|J_{I}\right|=|I|=1$. Finally, if $I$ consists of the first and the last row, then, by Claim 1, the two ones are in different columns, so $\left|J_{I}\right|=|I|=2$. This proves Claim 2.

Then, it follows from Hall's theorem $([4,13])$, that the bipartite graph of $A$ contains a perfect matching, and there is a permutation matrix $P$ with $P \preceq_{p} A$ with the desired properties.

The theorem assures that each of the matrices $A_{1}$ and $A_{2}$ in Example 6.8 has a permutation matrix that is smaller in the pattern order.

7. Coda. In this final section, we briefly consider chains in the pattern-plus partial order or, for brevity, pp-chains, and then return briefly to SRMs. 
Observation 7.1. Let $n$ be odd. Then the maximum cardinality of a pp-chain is

(a) $\left|\frac{(n-1)^{2}}{8}\right|+1$ if $n \equiv 3 \bmod 4$, and

(b) $\left[\frac{(n-1)^{2}}{8}\right]$ if $n \equiv 1 \bmod 4$.

In both cases, equality is attained by the diamond $A S M D_{n}$ by deleting alternating cycles of length 4 .

To see this, consider the diamond $\mathrm{ASM} A=\mathcal{D}_{n}$ in $\mathcal{A}_{n}$. It has the maximum number of nonzeros in that class; $A$ has $(1 / 4)(n-1)^{2}$ negative entries (and $n$ more than this positive entries). Also, in an pp-chain every matrix is obtained from the previous matrix by removing an alternating cycle, and this cycle has at least 2 negative entries. Thus, an upper bound on the cardinality of a pp-chain in $\mathcal{A}_{n}$ is

$$
\left\lfloor(1 / 2)(1 / 4)(n-1)^{2}\right\rfloor+1=\left\lfloor(n-1)^{2} / 8\right\rfloor+1 .
$$

First suppose that $n \equiv 3 \bmod 4$. We only illustrate a construction for $n=11$ which generalizes for all $n \equiv 3 \bmod 4:$

\begin{tabular}{|c|c|c|c|c|c|c|c|c|c|c|}
\hline & & & & & 1 & & & & & \\
\hline & & & & $a$ & $-a$ & 1 & & & & \\
\hline & & & 1 & $-a$ & $a$ & $-b$ & $b$ & & & \\
\hline & & $c$ & $-c$ & $d$ & $-d$ & $b$ & $-b$ & 1 & & \\
\hline & 1 & $-c$ & $c$ & $-d$ & $d$ & $-e$ & $e$ & $-f$ & $f$ & \\
\hline 1 & $-g$ & $g$ & $-h$ & $h$ & -1 & $e$ & $-e$ & $f$ & $-f$ & 1 \\
\hline & $g$ & $-g$ & $h$ & $-h$ & $i$ & $-i$ & $j$ & $-j$ & 1 & \\
\hline & & 1 & $-k$ & $k$ & $-i$ & $i$ & $-j$ & $j$ & & \\
\hline & & & $k$ & $-k$ & $l$ & $-l$ & 1 & & & \\
\hline & & & & 1 & $-l$ & $l$ & & & & \\
\hline & & & & & 1 & & & & & \\
\hline
\end{tabular}

where $a=b=c=d=e=f=g=h=i=j=k=l=1$.

Now suppose that $n \equiv 1 \bmod 4$. Then row $(n+1) / 2$ contains $(n+1) / 21$ 's and $(n-1) / 2(-1)$ 's. Then considering row $(n-1) / 2$ and row $(n+3) / 2$, we see that there do not exist pairwise disjoint alternating cycles of length 4 that include all the -1 's. It follows that the value given by (a) cannot be attained in this case and hence the value in (b) is an upper bound. Again we only illustrate a construction for $n=9$ to achieve the value in (b) which generalizes for all $n \equiv 1 \bmod 4$ :

$\left.\begin{array}{r|r|r|r|r|r|r|r|r} & & & & 1 & & & & \\ \hline & & & a & -a & 1 & & & \\ \hline & & 1 & -a & a & -b & b & & \\ \hline 1 & c & -c & d & -d & b & -b & 1 & \\ \hline & 1 & -f & f & -g & g & -e & e & \\ \hline & & f & -f & g & -g & 1 & & \\ \hline & & & 1 & -1 & 1 & & & \\ \hline & & & & 1 & & & & \end{array}\right]$

where $a=b=c=d=e=f=g=1$. 
Electronic Journal of Linear Algebra, ISSN 1081-3810

A publication of the International Linear Algebra Society

Volume 37, pp. 613-639, September 2021.

OBservation 7.2. Let $n$ be even. Then the maximum cardinality of a pp-chain is $\frac{(n-2)(n-4)}{4}+1$.

To see this, again consider the diamond $D_{n}$ which, now because $n$ is even (so $(n-1)$ is odd) has $(n-2)(n-4) / 2$ negative entries. Rows $(n / 2)$ and $((n+2) / 2)$ have $(n-1)$ nonzeros of which $(n-2) / 2$ are -1 's. If there were pairwise disjoint alternating cycles of length 4 that included all the -1 s of $D_{n}$, then the -1 's in each of these rows would have to be paired up and each pair in an alternating cycle of length 4 . It is easy to see that this is impossible.

There are alternating cycles of length 4 that contain all the -1 s and hence the maximum cardinality of a chain is as given. We illustrate a construction for $n=10$ :

$\left.\begin{array}{r|r|r|r|r|r|r|r|r|r} & & & & 1 & & & & & \\ \hline & & & a & -a & 1 & & & & \\ \hline & & 1 & -a & a & -b & b & & & \\ \hline & c & -c & d & -d & b & -b & 1 & & \\ \hline 1 & -c & c & -d & d & -e & e & -f & f & \\ \hline & 1 & -g & g & -1 & e & -e & f & -f & 1 \\ \hline & & g & -g & 1 & -1 & h & -h & 1 & \\ \hline & & & 1 & -i & i & -h & h & & \\ \hline & & & & i & -i & 1 & & & \\ \hline & & & & & 1 & & & & \end{array}\right]$,

where $a=b=c=d=e=f=g=h=i=1$. Since there are only two -1 's that are not part of the alternating cycles of length 4 , the number 9 of these alternating cycles is maximum.

We now briefly turn to the set $\mathcal{S}_{m, n}$ of $m \times n$ SRMs.

For a given $m \times n \operatorname{SRM} A$ define $t(A)$ as the minimum $t$ such that $A$ is a submatrix of a $t \times t$ ASM. Also, for given $m$ and $n$, let

$$
t\left(\mathcal{S}_{m, n}\right)=\max \left\{t(A): A \in \mathcal{S}_{m, n}\right\}
$$

Similarly, define

$$
t\left(\mathcal{S}_{m, n}^{+}\right)=\max \left\{t(A): A \in \mathcal{S}_{m, n}^{+}\right\} \text {and } t\left(\mathcal{S}_{m, n}^{++}\right)=\max \left\{t(A): A \in \mathcal{S}_{m, n}^{++}\right\} \text {. }
$$

Thus, any normalized SRM of size $m \times n$ (with no zero rows) can be extended to an ASM by adding at most $t\left(\mathcal{S}_{m, n}\right)$ lines.

THEOREM 7.3. Let $m$ and $n$ be positive integers with $m \leq n$. Let $A \in \mathcal{S R}_{m, n}^{+}$have $m-k$ zero rows where $0 \leq k<m$. Then $t(A) \leq 2 n+m-2 k$.

Proof. Suppose that $A$ contains rows with $l_{1}, l_{2}, \ldots, l_{k} 1$ 's, respectively, where the $l_{i}$ are positive (but the 1 's in a row need not be consecutive), and since every column contains a $1, l_{1}+l_{2}+\cdots+l_{k}=n$. With each row of $A$ with $l_{i} \geq 11$ 's we can build a $\left(2 l_{i}-1\right) \times\left(2 l_{i}-1\right)$ diamond ASM by inserting $\left(l_{i}-1\right)$ new columns and $2\left(l_{i}-1\right)$ new rows. The number of nonzero rows of the resulting matrix is $k+\sum_{i=1}^{k} 2\left(l_{i}-1\right)=2 n-k$; the number of columns equals $n+\sum_{i=1}^{k}\left(l_{i}-1\right)=n+n-k=2 n-k$. This gives a $p \times p$ ASM $A^{\prime}$ with $p=2 n-k$. This is clearly the minimum if $A$ does not have any zero rows, that is, if $k=m$.

If $k<m$, so that $A$ has $m-k$ rows of all 0's, then we can add $m-k$ new columns which are all zero except that the intersection with these $m-k$ rows is $I_{k}$ thereby giving a $(2 n+m-2 k) \times(2 n+m-2 k)$ $\operatorname{ASM} A^{*}$. 
We note the following. If there are zero rows of $A$, we can use them in the building of the ASM to construct $A^{*}$, thereby decreasing the size of $A^{*}$. We illustrate this in the next example.

EXAMPLE 7.4. Consider the $5 \times 6 \mathrm{SRM}$

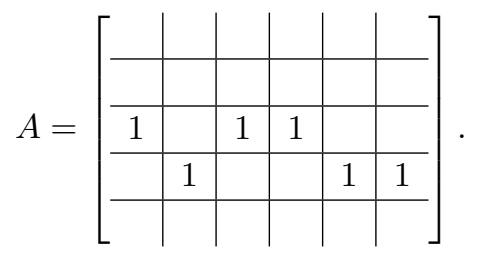

Then we construct the $10 \times 10 \mathrm{ASM}$

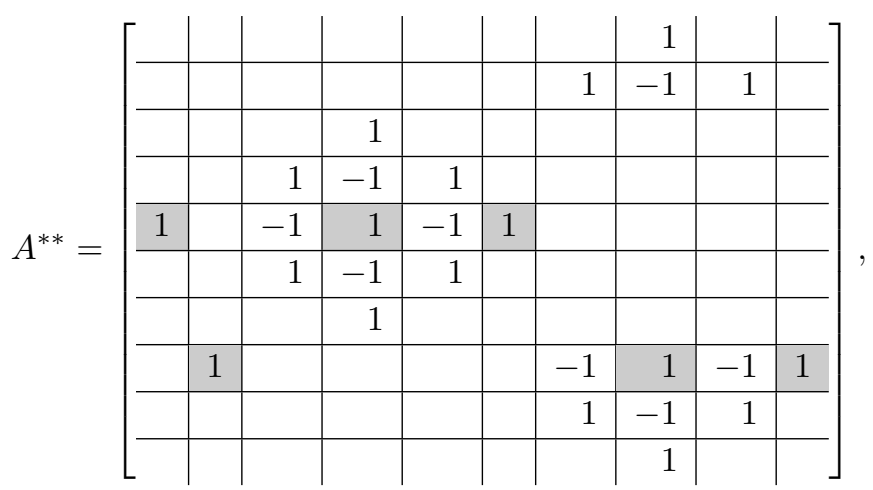

containing $A$ as a submatrix. Here we have taken advantage of zero rows of $A$ in inserting the appropriate diamond ASMs.

In particular, when $m=n$, since there are no zero rows, every matrix in $\mathcal{S R}_{n, n}$ is a permutation matrix and then clearly $t\left(\mathcal{S}_{n, n}^{+}\right)=n=2 n-n$. We remark that the ASM constructed in the proof of Theorem 7.3, after suitable line permutations, is a direct sum of diamond ASMs.

EXAMPLE 7.5. The construction in the previous proof is illustrated in two cases:

(i) $m=1, n=3, t\left(\mathcal{S}_{1,3}^{+}\right)=5$ :

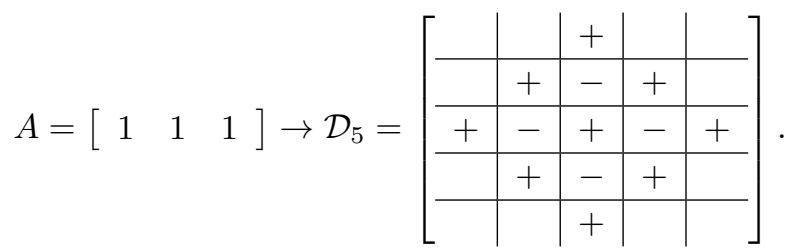

(ii) $m=2, n=5, t\left(\mathcal{S}_{2,5}^{+}\right)=8$ :

$$
A=\left[\begin{array}{lllll}
1 & 0 & 0 & 1 & 1 \\
0 & 1 & 1 & 0 & 0
\end{array}\right] \rightarrow B=
$$

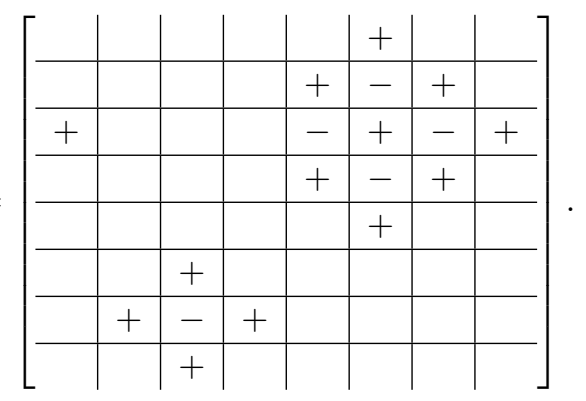


We conclude with some discussion concerning further research.

(i) In Theorem 5.12, we characterized the cover relation in the pattern-plus partial order, and in Theorem 6.7 we characterized the permutation matrices that are maximal in the pattern-plus partial order. The analogues of these characterizations for the pattern partial order have eluded us thus far.

(ii) Theorem 6.4 asserts that the pattern and pattern-plus partial orders have the same minimal elements. Theorem 6.9 concerns the interesting class $\mathcal{A}_{n}^{(3)}$ of ASMs in which all rows and columns other than the first and last have exactly three nonzeros. An ASM that covers a permutation matrix in the pattern partial order may necessarily belong to this class. Regarding this, it is interesting to ask which signed permutation matrices with at least one -1 can be extended to an ASM by changing some 0 's to \pm 1 and if this can be done, is there always such an ASM in $\mathcal{A}_{n}^{(3)}$ ?

(iii) Finally, there are several natural questions for SRMs, along the lines of our results for ASMs in the second part of the paper.

Acknowledgments. The authors thank a referee for careful reading of the paper and useful comments.

\section{REFERENCES}

[1] J.C. Aval, Keys and alternating sign matrices. Smin. Lothar. Comb. 59:B59F, 2007/2010.

[2] R.E. Behrend and V.A. Knight. Higher spin alternating sign matrices. Electron. J. Combin. 14:\#1, 2007.

[3] D. Bressoud. Proofs and Confirmations. The Story of the Alternating Sign Matrix Conjecture. MAA Spectrum, Math. Assoc. America. Cambridge Univ. Press, Washington, DC, 1994.

[4] R.A. Brualdi and H.J. Ryser. Combinatorial Matrix Theory. Cambridge University Press, 1991.

[5] R.A. Brualdi. Combinatorial Matrix Classes. Cambridge University Press, 2006.

[6] R.A. Brualdi, K.P. Kiernan, S.A. Meyer, and M. W. Schroeder. Patterns of alternating sign matrices. Linear Algebra Appl. 438:3967-3990, 2013.

[7] R.A. Brualdi and H.K. Kim. Completions of alternating sign matrices. Graphs Combin. 3:507-522, 2015.

[8] R.A. Brualdi and M.W. Schroeder. Alternating sign matrices and their Bruhat order. Discrete Math. 340:1996-2019, 2017.

[9] R.A. Brualdi and G. Dahl. Alternating sign matrices, extensions and related cones. Adv. Appl. Math. 86:19-49, 2017.

[10] R.A. Brualdi and G. Dahl. Alternating sign matrices and hypermatrices, and a generalization of Latin squares. Adv. Appl. Math. 95:116-151, 2018.

[11] R.A. Brualdi and G. Dahl. Sign-restricted matrices of 0's, 1's, and -1's. Linear Algebra Appl. 615:77-103, 2021.

[12] J. Engbers and A. Hammett. Trivial meet and join within the lattice of monotone triangles. Electron. J. Combin. 21(3), Research paper 31, 15pp, 2014.

[13] L. Lovász and M.D. Plummer. Matching Theory. North-Holland, Amsterdam, $1986 .$.

[14] A. Lascoux and M.-P.Schützenberger. Treillis et bases des groupes de Coxeter, Electron. J. Combin. 3:\# R27, 1996.

[15] W.H. Mills, D.P. Robbins, and H. Rumsey. Alternating-sign matrices and descending plane partitions. J. Combin. Theory Ser. A 34:340-359, 1983.

[16] A. Schrijver. Theory of Linear and Integer Programming. Wiley-Interscience, Chichester, 1986.

[17] S. Solhjem and J. Striker. Sign matrix polytopes from Young tableaux. Linear Algebra Appl. 574:84-122, 2019.

[18] J. Striker. The alternating sign matrix polytope. Electron. J. Combin. 16(1), Research paper 41, 15 pp, 2009. 